COMMUNICATIONS IN

ANALYSIS AND GEOMETRY

Volume 4, Number 2, 207-259, 1996

\title{
Uniformizations of modular curves
}

\author{
Hershel M. Farkas, YaACOV Kopeliovich, AND IrWin Kra ${ }^{1}$
}

\section{Introduction.}

In a recent paper [7], two of the authors ${ }^{2}$ of this paper used the theory of theta constants with rational characteristics to study automorphic forms and functions for the prime level principal congruence subgroups $\Gamma(k)$ of the modular group $\Gamma$. A byproduct of this investigation was a new cubic theta constant identity, and explicit mappings of the Riemann surfaces $\mathbb{H}^{2} / \Gamma(k)$ onto punctured spheres for the cases $k=3,5^{3}$. In [5] this theory is extended from primes to arbitrary positive integers. One of the main tools used is the association of theta characteristics to the cusps of the group. This again leads to new theta constant identities and explicit construction of covering maps for the cases $k=4$ and 6 . In this second paper, much of the theory is extended to two other families of groups: $G(k)$ and $\Gamma_{0}(k)$.

The cubic identity in [7] led to a paper [3] where a general $k^{\text {th }}$ power identity was derived with $k$ any odd positive integer. The techniques used in [7] also gave rise to identities of Ramanujan type [4]. The main theorem of [4] is a tool for the construction of quartic three term theta constant identities which we will, among other things, interpret in this paper.

Many facts are known about the congruence subgroups of the modular group and the Riemann surfaces they define. For example, we learn from the theory of group representations that for every odd prime $k>3$ (see [11]) the finite group $\Gamma / \Gamma(k)$ admits a faithful representations as a subgroup of $\mathrm{GL}\left(\frac{k-1}{2}, \mathbb{C}\right)$; but no lower rank will do. We (almost) realize these representations using concrete Hilbert spaces of functions. We only get a representation $\rho$ of $\Gamma(k)$ into PGL $\left(\frac{k-1}{2}, \mathbb{C}\right)$; however, the image group, $\rho(\Gamma(k))$

\footnotetext{
${ }^{1}$ Research by IK supported in part by NSF Grant DMS 9204092. Research by HF sponsored in part by the Edmund Landau Center for Research in Mathematical Analysis supported by the Minerva Foundation (Germany).

${ }^{2}$ YK thanks R. Livne and A. Reznikov for the time and effort they devoted to a discussion of aspects of this work.

${ }^{3}$ Notation from [7] and [5].
} 
consists of unitary matrices. In particular, for odd $k$, we shall obtain an explicit holomorphic map of $\mathbb{H}^{2} / \Gamma(k)$ into $\mathbf{P} \mathbb{C}^{\frac{k-3}{2}}$, and a group of projective transformations of this space that is, for $k$ prime, an isomorphic image of $\Gamma / \Gamma(k)$, the automorphism group of $\mathbb{H}^{2} / \Gamma(k)$.

The novelty of our approach is in that we are able to connect the representation of $\Gamma / \Gamma(k)$ to the geometry of the image of the compactification of the surface $\mathbb{H}^{2} / \Gamma(k)$ in $\mathbf{P} \mathbb{C}^{\frac{k-3}{2}}$. While the representations in these dimensions were known previously there was no connection with the geometry of the curve. The very explicit form of the representation allows us, for example, to use the punctures on $\mathbb{H}^{2} / \Gamma(k)$ to obtain an ideal triangulation of this surface that contains significant geometric information about the surfaces represented by the congruence subgroups. Previously all that one knew was that the group operated transitively on the punctures. The fact the group now acts as isometries in the projective space gives much more information.

The functions which appear in our theory for $k=3,5$ are in fact the automorphic forms which appear in Ramanujan's theory of congruences for the partition function and the automorphic forms which appear in the celebrated Rogers Ramanujan identities. This suggests that the ideas whose exploration is begun here are candidates for a great deal of further development.

We give a partial description of the curve $\mathbb{H}^{2} / \Gamma(k)$ sitting in projective space $\mathbf{P} \mathbb{C}^{\frac{k-3}{2}}$ in terms of theta constant identities. We obtain, for $k$ prime, generators for the field of meromorphic functions on the compactification $\overline{\mathbb{H}^{2} / \Gamma(k)}$ of $\mathbb{H}^{2} / \Gamma(k)$. This theory begins with the case $k=5$; although in [7] we also obtained a representation of $\Gamma / \Gamma(3)$ as a group of fractional linear transformations. Our work, especially for the case $k=5$, overlaps, in part, with the study of Fricke-Klein [8, pg. 613, vol. I and pg. 383, vol. II]. See, in particular, [12] and [13] for classical work of F. Klein on related questions. Our methods and results are quite different and allow considerable generalization. We treated the case $k=5$ in some detail in [7]; we present an alternate development which leads naturally to the appearance of the regular icosahedron, and suggests generalizations of the Platonic solids to be investigated in subsequent papers. We study in detail the case $k=$ 7 obtaining the classical uniformization of the Klein surface, the unique (see [17] and [18] and the literature quoted there) surface of genus 3 with automorphism group of order 168. We also treat the cases $k=11,13$ and 9; in part to suggest future directions for our research. The main point of the paper is to illustrate how the theory of rational characteristics is useful in concrete computations. We are mostly interested in the study of the 
holomorphic functions on the upper half plane

$$
\tau \mapsto \theta[\chi]\left(0, k^{\prime} \tau\right)
$$

for a restricted class of characteristics $\chi$ and various positive integers $k^{\prime} \mid k$. Whereas [7] and [5] considered the case $k^{\prime}=1$, this paper deals with $k^{\prime}=k$. The more general case remains to be studied. We concentrate mostly on the case where $k$ is an odd prime ${ }^{4}$; however, in some instances we lay the groundwork for a forthcoming in-depth study of the composite case.

\section{A prescription for obtaining the equations of the modular curves.}

\subsection{The function field $\mathcal{K}\left(\overline{\mathbb{H}^{2} / \Gamma(k)}\right)$.}

The $\jmath$-function on $\mathbb{H}^{2}$ is invariant under $\Gamma$; hence certainly under $\Gamma(k)$. It defines a degree $k n(k)$ function on the compact Riemann surface $\overline{\mathbb{H}^{2} / \Gamma(k)}$ that is holomorphic on the punctured surface $\mathbb{H}^{2} / \Gamma(k)$. Choose any point $a \in$ $\mathbb{H}^{2}$ not fixed by an elliptic element of $\Gamma$. Let us choose a set of representatives

$$
a_{1}, \ldots, a_{k n(k)}
$$

for the orbit $\{(\Gamma / \Gamma(k))(a)\}$. Let $w$ be any meromorphic function on $\overline{\mathbb{H}^{2} / \Gamma(k)}$ produced as a ratio of theta constants. Such a function is regular (holomorphic and nonzero) on $\mathbb{H}^{2} / \Gamma(k)$. Assume that the $k n(k)$ values $\left\{w\left(a_{j}\right)\right\}$ are distinct. We now construct a second meromorphic function

$$
W=\sum_{j=1}^{k n(k)} \frac{1}{\left(w-w\left(a_{j}\right)\right)^{k_{j}}},
$$

where the positive integers $k_{j}$ are chosen so that the orders of the poles of $W$ are distinct. Then the arguments in the proof of [6, Proposition IV.11.7] show that $J$, the projection of $\jmath$ to $\overline{\mathbb{H}^{2}} / \Gamma(k)$, and $W$ (hence also $J$ and $w$ ) generate $\mathcal{K}\left(\overline{\mathbb{H}^{2} / \Gamma(k)}\right)$, the field of meromorphic functions on the compact Riemann surface $\overline{\mathbb{H}^{2} / \Gamma(k)}$. Since it is difficult to produce the function $w$, we turn to other methods to find generators for the function field $\mathcal{K}\left(\overline{\mathbb{H}^{2}} / \Gamma(k)\right)$.

\footnotetext{
${ }^{4}$ Throughout this paper $k$ is an integer greater than 1 . Unless otherwise stated, it represents a prime greater than 2 .
} 


\subsection{Primitive pairs.}

Let $M$ be a compact Riemann surface. Let $\mathfrak{M}^{*}$ be the set of analytic configurations (equivalence classes of convergent Puiseaux series). We are using the language and results from [6, §IV.11]. Let $z$ and $w$ be two nonconstant meromorphic functions on $M$ (thus elements of $\mathcal{K}(M)$ ). These two functions define a nonconstant holomorphic map

$$
\varphi: M \rightarrow \mathfrak{M}^{*}
$$

such that for all $x \in M$,

$$
w(x)=\operatorname{eval}(\varphi(x)) \text { and } z(x)=\operatorname{proj}(\varphi(x)) .
$$

We are interested in simple conditions that guarantee that the map $\varphi$ is injective (equivalently, that $z$ and $w$ form a primitive pair on $M$ or that they generate $\mathcal{K}(M))$.

Proposition 2.1. Let $z$ and $w$ be nonconstant meromorphic functions on $M$. If $\operatorname{deg} z$ is prime and proj is not injective on $\varphi(M)$, then $\varphi$ is injective (on $M$ ).

Proof. From the second equation in (2.1) we conclude that

$$
\operatorname{deg} z=(\operatorname{deg} \operatorname{proj})(\operatorname{deg} \varphi) .
$$

If proj is one-to-one, $\varphi(M)$ is (conformally equivalent to) the Riemann sphere; otherwise, $\operatorname{deg} \operatorname{proj}=\operatorname{deg} z$.

\subsection{Zeros and poles.}

Let $z$ and $w$ be nonconstant meromorphic functions on the compact Riemann surface $M$. The map $\varphi$ assigns to each point $P \in M$, the Puiseaux series of $w$ at $P$ in terms of $z$. Assume we know the divisor $(z)$ and the leading terms of the Laurent series expansion of $w$ at the support of $(z)$. Equation (2.1) or equivalently (2.2) is then a useful tool for determining when $\varphi$ is injective. From the divisor $(z)$ we easily compute $\operatorname{deg} z$. We compute deg proj as the number of zeros (counting multiplicities) of the function proj on $\varphi(M)$. Let $P_{1}, \ldots, P_{r}$ be a complete list of the distinct 
zeros of $z$. Assume that $w$ weakly separates these points in the sense that for $1 \leq i<j \leq r$ either

$$
w\left(P_{i}\right) \neq w\left(P_{j}\right) \text { or } b_{w}\left(P_{i}\right) \neq b_{w}\left(P_{j}\right)
$$

and for each $i$,

$$
\left(b_{z}\left(P_{i}\right)+1, b_{w}\left(P_{i}\right)+1\right)=1 .
$$

The first condition (2.3) tells us that the $r$ Puiseaux series $\left\{\varphi\left(P_{i}\right) ; i=\right.$ $1, \ldots, r\}$ are distinct; the second (2.4) allows us to compute the ramification number of proj at each $P_{i}$ (from purely local data). We conclude that under these hypotheses,

$$
\operatorname{deg} \operatorname{proj}=\sum_{i=1}^{r}\left(b_{z}\left(P_{i}\right)+1\right)=\operatorname{deg} z ;
$$

which shows, of course, that $\varphi$ is injective whenever (2.3) and (2.4) are satisfied.

\subsection{The degree of the map $\varphi$.}

Let $t$ be a local coordinate vanishing at $P_{1}$ such that in a neighborhood of $P_{1}$,

$$
z(t)=t^{\alpha}
$$

for the positive integer $\alpha=\operatorname{ord}_{P_{1}} z$. Then $\varphi\left(P_{1}\right)$ is a Puiseaux series of the form

$$
w(z)=\sum_{j=\beta}^{\infty} c_{j} z^{\frac{j}{\alpha}}, \beta=\operatorname{ord}_{P_{1}} w .
$$

It now easily follows that if $\varphi(P)=\varphi\left(P_{1}\right)$ for some $P \in M$, then $z$ vanishes at $P$ and hence $P=P_{i}$ for some $i, i=1, \ldots, r$. If, for example,

$$
\operatorname{ord}_{P_{i}} w \neq \operatorname{ord}_{P_{1}} w, i=2, \ldots, r
$$

and

$$
\left(\operatorname{ord}_{P_{i}} w, \operatorname{ord}_{P_{i}} z\right)=1, i=1, \ldots, r
$$

then $\operatorname{deg} \varphi=1$. 


\section{Old theta identities.}

We list some basic identities among theta constants that we will use repeatedly. All of them have analogues for theta functions. For all characteristics $\left[\begin{array}{c}\epsilon \\ \epsilon^{\prime}\end{array}\right] \in \mathbb{R}^{2}$

$$
\theta\left[\begin{array}{c}
-\epsilon \\
-\epsilon^{\prime}
\end{array}\right]=\theta\left[\begin{array}{c}
\epsilon \\
\epsilon^{\prime}
\end{array}\right]
$$

and if we also fix a pair of integers $m$ and $n$, then

$$
\theta\left[\begin{array}{c}
\epsilon+2 m \\
\epsilon^{\prime}+2 n
\end{array}\right]=\exp \{\pi \imath \epsilon n\} \theta\left[\begin{array}{c}
\epsilon \\
\epsilon^{\prime}
\end{array}\right] .
$$

For all characteristics $\left[\begin{array}{c}\epsilon \\ \epsilon^{\prime}\end{array}\right] \in \mathbb{R}^{2}$, all positive integers $n$, and all $\tau \in \mathbb{H}^{2}$,

$$
\theta\left[\begin{array}{c}
\epsilon \\
\epsilon^{\prime}
\end{array}\right](0, \tau)=\sum_{l=0}^{n-1} \theta\left[\begin{array}{c}
\frac{2 l+\epsilon}{n} \\
n \epsilon^{\prime}
\end{array}\right]\left(0, n^{2} \tau\right) .
$$

Finally, for all characteristics $\chi=\left[\begin{array}{c}\epsilon \\ \epsilon^{\prime}\end{array}\right] \in \mathbb{R}^{2}$, all matrices $\gamma=\left[\begin{array}{cc}a & b \\ c & d\end{array}\right] \epsilon$ $\mathrm{SL}(2, \mathbb{Z})$, and all $\tau \in \mathbb{H}^{2}$,

$$
\theta[\chi](0, \gamma(\tau))=\kappa(\chi, \gamma)(c \tau+d)^{\frac{1}{2}} \theta[\chi \gamma](0, \tau),
$$

where

$$
\kappa(\chi, \gamma)=\exp 2 \pi \imath\left\{-\frac{1}{4}\left(a \epsilon+c \epsilon^{\prime}\right) b d-\frac{1}{8}\left(a b \epsilon^{2}+c d \epsilon^{2}+2 b c \epsilon \epsilon^{\prime}\right)\right\} \kappa(0, \gamma) .
$$

Note that the ambiguity of sign in $[7,(6)]$ is absorbed in the last term in the above product (since it is independent of $\chi$; but does depend on the choice of square root of $(c \tau+d)$ ).

We will use the quasi-periodicity of the theta function $[7,(1)]$

$$
\theta\left[\begin{array}{c}
\epsilon \\
\epsilon^{\prime}
\end{array}\right](z+n+m \tau, \tau)=\exp 2 \pi \imath\left\{\frac{n \epsilon-m \epsilon^{\prime}}{2}-m z-\frac{m^{2}}{2} \tau\right\} \theta\left[\begin{array}{c}
\epsilon \\
\epsilon^{\prime}
\end{array}\right](z, \tau),
$$


for all $n, m \in \mathbb{Z}$, all $z \in \mathbb{C}$, all $\tau \in \mathbb{H}^{2}$, and we will need to relate the value of the theta function (with a given characteristic) at a point of finite order on the torus to the value of a related theta constant [7, (2)]

$$
\theta\left[\begin{array}{c}
\epsilon \\
\epsilon^{\prime}
\end{array}\right](\alpha \tau, \tau)=\exp -\pi \imath\left\{\alpha^{2} \tau+\alpha \epsilon^{\prime}\right\} \theta\left[\begin{array}{c}
\epsilon+2 \alpha \\
\epsilon^{\prime}
\end{array}\right](0, \tau)
$$

for all $\alpha \in \mathbb{R}$.

\section{Primitive invariant automorphic forms.}

\subsection{A Hilbert space of modified theta constants.}

Let $V(k)$ be the finite dimensional vector space of holomorphic functions on $\mathbb{H}^{2}$ spanned by the modified theta constants

$$
\tau \mapsto \varphi_{l}(\tau)=\theta\left[\chi_{l}\right](0, k \tau),
$$

where the characteristic ${ }^{5} \chi_{l}=\left[\begin{array}{c}\frac{2 l+1}{k} \\ 1\end{array}\right], l=0, \ldots, \frac{k-3}{2}$, for odd $k$ and $\chi_{l}=\left[\begin{array}{c}\frac{2 l}{k} \\ 0\end{array}\right], l=0, \ldots, \frac{k}{2}$, for even $k$. Define $V_{o}(k)$ to be the linear span of those modified theta constants $\varphi_{l}$ for which the characteristic $\chi_{l} \in X_{o}(k)^{6}$.

Lemma 4.1. For each $k \in \mathbb{Z}, k>1$,

$$
\operatorname{dim} V(k)=\frac{k-1}{2} \text { for odd } k \text { and } \operatorname{dim} V(k)=\frac{k}{2}+1 \text { for even } k .
$$

Proof. Note that in terms of the local coordinate described below

$$
\operatorname{ord}_{\infty} \varphi_{l}=\frac{(2 l+1)^{2}}{8} \text { for odd } k \text { and } \operatorname{ord}_{\infty} \varphi_{l}=\frac{l^{2}}{2} \text { for even } k .
$$

${ }^{5}$ The characteristic $\chi_{0}$ defined above and the characteristic $\chi_{o}$ of [5] are related. For odd $k, \chi_{0} A$ is equivalent to $\chi_{o}$.

${ }^{6}$ This symbol is defined in [5]. For the special case under consideration here it means that $(2 l+1, k)=1$ if $k \equiv 1 \bmod 2,(2 l, k)=2$ and $l$ is even if $k \equiv 2 \bmod 4$, and $(2 l, k)=2$ and $l$ is odd if $k \equiv 0 \bmod 4$. 
The modular group $\Gamma$ acts on $V(k)$ (Lemma, 4.2). It does not act on $V_{o}(k)$ (Remark 3). We record for future use the useful observations concerning the Fourier series expansions of the modified theta constants have. In terms of $\zeta=\exp \left\{\frac{2 \pi \imath \tau}{k}\right\}, \tau \in \mathbb{H}^{2}$, they are given by

$$
\begin{aligned}
& \Phi_{l}(\zeta)=\zeta^{\frac{(2 l+1)^{2}}{8}}\left(\exp \left\{\frac{\pi \imath(2 l+1)}{2 k}\right\}\right. \\
&\left.+\exp \left\{\frac{\pi \imath(2 l+1-2 k)}{2 k}\right\} \zeta^{k\left(\frac{k-2 l-1}{2}\right)}+\ldots\right) \\
& \quad=\exp \left\{\frac{\pi \imath(2 l+1)}{2 k}\right\} \zeta^{\frac{(2 l+1)^{2}}{8}}\left(1-\zeta^{k\left(\frac{k-2 l-1}{2}\right)}+\ldots\right)
\end{aligned}
$$

and hence (for $l^{\prime}$ also in $\mathbb{Z}$ with $0 \leq l^{\prime} \leq \frac{k-3}{2}$ )

$$
\begin{aligned}
\frac{\Phi_{l}}{\Phi_{l^{\prime}}}(\zeta)=\zeta^{\frac{\left(l+l^{\prime}+1\right)\left(l-l^{\prime}\right)}{2}} \exp \left\{\frac{\pi \imath\left(l-l^{\prime}\right)}{k}\right\} & \\
& \times\left(1-\zeta^{k\left(\frac{k-2 l-1}{2}\right)}+\zeta^{k\left(\frac{k-2 l^{\prime}-1}{2}\right)}+\ldots\right)
\end{aligned}
$$

Lemma 4.2. For each $\gamma \in \Gamma$, the linear operator on functions

$$
\gamma_{*}: f \mapsto(f \circ \gamma)\left(\gamma^{\prime}\right)^{1 / 4}
$$

maps $V(k)$ onto itself.

Proof. In defining the operator $\gamma_{*}$, we are making a specific choice for $\left(\gamma^{\prime}\right)^{\frac{1}{4}}$. If we view $\gamma$ as an element of $\operatorname{SL}(2, \mathbb{C})$ (respectively, $\operatorname{PSL}(2, \mathbb{C})$ ), the operator involves a choice of square (fourth) root of unity. Since for each $\gamma_{1}$ and $\gamma_{2}$ in $\Gamma$,

$$
\left(\gamma_{1} \circ \gamma_{2}\right)_{*}=c\left(\gamma_{2}\right)_{*} \circ\left(\gamma_{1}\right)_{*}
$$

where $c$ is a fourth root of unity, it suffices to show that the operators defined by the generators $B$ and $A$ of $\Gamma$ preserve $V(k)$.

Assume $k$ is odd. We start with the generator $B$. For $\tau \in \mathbb{H}^{2}$, we find from the transformation formulae [7, (6) and (3)] (or (3.4) and (3.2), above) 
for theta constants that

$$
\begin{aligned}
& \theta\left[\begin{array}{c}
\frac{2 l+1}{k} \\
1
\end{array}\right](0, k(\tau+1))= \\
& =\kappa\left(\left[\begin{array}{c}
\frac{2 l+1}{k} \\
1
\end{array}\right], B^{k}\right) \theta\left[\begin{array}{c}
\frac{2 l+1}{k} \\
1+2 l+2+(k-1)
\end{array}\right](0, k \tau) \\
& =c(B, k) \exp \left\{\frac{\pi l}{k}\left(l^{2}+l\right)\right\} \theta\left[\begin{array}{c}
\frac{2 l+1}{k} \\
1
\end{array}\right](0, k \tau)
\end{aligned}
$$

that is,

$$
B_{*}\left(\varphi_{l}\right)=c(B, k) \exp \left\{\frac{\pi \imath}{k}\left(l^{2}+l\right)\right\} \varphi_{l}, c(B, k)=\exp \left\{\frac{\pi \imath}{4 k}\right\} .
$$

In the above and subsequent formulae $c(\gamma, k)$ is a constant of absolute value 1 that depends only on the transformation $\gamma \in \Gamma$ and the integer $k$. At times we specify the constant for future applications.

The situation for the second generator $A$ of $\Gamma$ is both more complicated and more interesting. Again for $\tau \in \mathbb{H}^{2}$,

$$
\begin{gathered}
\tau^{-\frac{1}{2}} \theta\left[\begin{array}{c}
\frac{2 l+1}{k} \\
1
\end{array}\right]\left(0, \frac{-1}{\frac{\tau}{k}}\right)=c(A, k) \exp \left\{\frac{\pi l l}{k}\right\} \theta\left[\begin{array}{c}
1 \\
\frac{2 l+1}{k}
\end{array}\right]\left(0, \frac{\tau}{k}\right) \\
=c(A, k) \exp \left\{\frac{\pi \imath l}{k}\right\} \sum_{j=0}^{k-1} \theta\left[\begin{array}{c}
\frac{2 j+1}{k} \\
2 l+1
\end{array}\right](0, k \tau), c(A, k)=\frac{1}{\sqrt{\imath k}} \exp \left\{\pi \imath \frac{1}{2 k}\right\} .
\end{gathered}
$$

We have used (3.3) to obtain the last of the above equalities. Now it is obvious that the last expression can be rewritten as

$$
\sum_{j=0}^{\frac{k-3}{2}} c_{j} \theta\left[\begin{array}{c}
\frac{2 j+1}{k} \\
1
\end{array}\right](0, k \tau) .
$$

In the last expression the numbers $c_{j}$ (that depend on $A, k$, and $j$ ) are easily computed. We can rewrite our last equation as

$$
A_{*}\left(\varphi_{l}\right)=\frac{1}{\sqrt{\imath k}} \exp \left\{\pi \imath \frac{1}{2 k}\right\} \sum_{j=0}^{\frac{k-3}{2}} A_{l j} \varphi_{j} .
$$

Lengthy but routine calculations show that

$$
A_{l j}=\exp \left\{2 \pi \imath \frac{l(j+1)}{k}\right\}+\exp \left\{-\pi \imath \frac{1}{k}\right\} \exp \left\{-2 \pi \imath \frac{(l+1) j}{k}\right\} .
$$


Define $\mathcal{A}$ as the $\frac{k-1}{2} \times \frac{k-1}{2}$ matrix whose $l j$ entry, $l, j=0, \ldots, \frac{k-3}{2}$, is $\left(\exp \left\{\pi \imath \frac{1}{2 k}\right\}\right) A_{l j}$. If for $z \in \mathbb{H}^{2}$, we choose the branch of $z^{\frac{1}{2}}$ to have positive imaginary part, then we conclude that $A_{*}^{2}=-\imath I$. In particular, we see that $A_{*}^{-1}=\imath A_{*}$. If we identify the operator $A_{*}$ with the matrix that represents it with respect to the basis $\left\{\varphi_{0}, \ldots, \varphi_{\frac{k-3}{2}}\right\}$, then we see that

$$
A_{*}=\frac{1}{\sqrt{\imath k}} \mathcal{A} \text { and } \mathcal{A}^{2}=k I \text {. }
$$

The matrix $\mathcal{A}$ is obviously hermitian (that is, $\mathcal{A}={ }^{t} \overline{\mathcal{A}}$ ). It now follows easily that $A_{*}$ is unitary (that is, $A_{*}^{-1}={ }^{t} \overline{A_{*}}$ ). The matrix representing the operator $B_{*}$ is obviously unitary. Hence for all $\gamma \in \Gamma$, the matrix representing $\gamma_{*}$ is unitary ${ }^{7}$.

For even $k$, the formulae corresponding to (4.3) and (4.4) are

$$
\theta\left[\begin{array}{c}
\frac{2 l}{k} \\
0
\end{array}\right](0, k(\tau+1))=c(B, k) \exp \left\{\pi \imath \frac{l^{2}}{k}\right\} \theta\left[\begin{array}{c}
\frac{2 l}{k} \\
0
\end{array}\right](0, k \tau),
$$

and

$$
\tau^{-\frac{1}{2}} \theta\left[\begin{array}{c}
\frac{2 l}{k} \\
0
\end{array}\right]\left(0, \frac{-1}{\frac{\tau}{k}}\right)=\sum_{j=0}^{\frac{k}{2}} c_{j} \theta\left[\begin{array}{c}
\frac{2 j}{k} \\
0
\end{array}\right](0, k \tau),
$$

respectively.

Remark 1. The transformation formula for $B_{*}$ shows that $\varphi_{l}$ is an eigenvector for $B_{*}$; this formula and the one for $A_{*}$ will facilitate computations of the divisors of certain $\Gamma(k)$-automorphic functions, as well as the images of the punctures on $\mathbb{H}^{2} / \Gamma(k)$ under a holomorphic map $\Phi: \overline{\mathbb{H}^{2} / \Gamma(k)} \rightarrow \mathbf{P} \mathbb{C}^{\frac{k-3}{2}}$ that will be defined in $\S 4.3$.

\subsection{A second Hilbert space of modified theta constants.}

For $k \in \mathbb{Z}^{+}$, let $W(k)$ be the finite dimensional vector space of holomorphic functions on $\mathbb{H}^{2}$ spanned by the modified. theta constants

$$
\tau \mapsto \psi_{l}(\tau)=\exp \left\{\pi \imath \frac{1+2 l}{2 k}\right\} \theta\left[\chi_{l}\right]\left(0, \frac{\tau}{k}\right),
$$

${ }^{7}$ The next subsection contains a second proof of this assertion. The next proposition gives an orthogonal basis for $V(k)$. 
where the characteristic $\chi_{l}=\left[\begin{array}{c}1 \\ \frac{2 l+1}{k}\end{array}\right], l=0, \ldots, \frac{k-3}{2}$, for odd $k$ and $\chi_{l}=\left[\begin{array}{c}0 \\ \frac{2 l}{k}\end{array}\right], l=0, \ldots, \frac{k}{2}$, for even $k$. Define $W_{o}(k)$ to be the linear span of those modified theta constants $\psi_{l}$ for which the characteristic $\chi_{l} \in X_{o}(k)$. Let us asume for the remainder of this subsection that $k$ is an odd integer. The appropriate Fourier series expansion for these functions $\psi$ is

$$
\begin{aligned}
\Psi_{l}(\zeta)=2 \exp \{\pi \imath & \left.\frac{1+2 l}{2 k}\right\} \\
& \times \zeta^{\frac{1}{8}}\left(\cos \left\{\frac{\pi(2 l+1)}{2 k}\right\}+\cos \left\{\frac{3 \pi(2 l+1)}{2 k}\right\} \zeta+\ldots\right) .
\end{aligned}
$$

The spaces $V(k)$ and $W(k)$ become Hilbert spaces under the Petersson pairing

$$
<\varphi, \psi>=\iint_{\mathbb{H}^{2} / \Gamma(k)}(\Im z)^{-\frac{3}{2}} \varphi(z) \overline{\psi(z)}\left|\frac{d z \overline{d z}}{2}\right|,
$$

$\varphi$ and $\psi$ both in either $V(k)$ or $W(k)$. An easy calculation now shows that for each $\gamma \in \Gamma$, the linear operator $\gamma_{*}$ is unitary; that is,

$$
<\gamma_{*}(\varphi), \gamma_{*}(\psi)>=<\varphi, \psi>
$$

for all $\gamma \in \Gamma$ and all $\varphi$ and $\psi$ both in either $V(k)$ or $W(k)$. Since $A_{*}(V(k))=$ $W(k)$ (because

$$
A_{*}\left(\varphi_{l}\right)=\frac{1}{\sqrt{\imath k}} \psi_{l}
$$

for $\left.l=0, \ldots, \frac{k-3}{2}\right)$, and $A_{*}$ preserves $V(k)$, we conclude that $V(k)=W(k)$.

Proposition 4.3. For each odd prime $k$, the $\frac{k-1}{2}$ functions $\left\{\varphi_{0}, \ldots, \varphi_{\frac{k-3}{2}}\right\}$ form an orthogonal basis for the Hilbert space $V(k)$.

Proof. We compute for integers $l$ and $l^{\prime}$ with $0 \leq l^{\prime}<l \leq \frac{k-3}{2}$,

$$
<\varphi_{l}, \varphi_{l^{\prime}}>=<B_{*}\left(\varphi_{l}\right), B_{*}\left(\varphi_{l^{\prime}}\right)>=\exp \left\{\frac{\pi \imath}{k}\left(l^{2}+l-l^{\prime 2}-l^{\prime}\right)\right\}<\varphi_{l}, \varphi_{l^{\prime}}>.
$$

Since $k$ does not divide $\left(l-l^{\prime}\right)\left(l+l^{\prime}+1\right)$ we conclude that

$$
\exp \left\{\frac{\pi \imath}{k}\left(l^{2}+l-l^{2}-l^{\prime}\right)\right\} \neq 1
$$


and hence

$$
<\varphi_{l}, \varphi_{l^{\prime}}>=0 .
$$

We have reproven, in this setting, the well known fact that eigenvectors belonging to distinct eigenvalues are orthogonal.

\subsection{Projective representation of Aut $\mathbb{H}^{2} / \Gamma(k)$.}

It is a consequence of (4.2) that we can define a homomorphism of $\Gamma$ into the group of linear transformations of $V(k)$ modulo nonzero multiples of the identity by sending $\gamma \in \Gamma$ to the linear operator $\gamma_{*}^{-1}$. We have hence a well defined homomorphism of $\Gamma$ into the projective linear transformations of $\mathbf{P} V(k)$, projectivized $V(k)$, which can be identified, for odd $k$, with $\mathbf{P} \mathbb{C}^{\frac{k-3}{2}}$. Let

$$
\Theta: \Gamma \rightarrow \text { Aut } \mathbf{P} V(k)
$$

denote this homomorphism. We have already observed that each $\varphi_{l}$ is an eigenvector for the operator $B_{*}$. Except for $k=3$, the operator $B_{*}$ has two or more distinct eigenvalues. Hence $B$ is not in the kernel of $\Theta$ for $k \neq 3$. We claim that $\Gamma(k)$ is in the kernel of $\Theta$ for odd $k$. We start with a slightly more general situation and use a "cheap (apparently classical) trick." Let $\gamma \in \Gamma_{o}(k)$ be represented by the matrix $\left[\begin{array}{ll}a=a(\gamma) & b=b(\gamma) \\ c=c(\gamma) & d=d(\gamma)\end{array}\right]$ in $\mathrm{SL}(2, \mathbb{Z})$ (hence $c$ is a multiple of $k$ ). Therefore for $\tau \in \mathbb{H}^{2}, k \gamma(\tau)=k \frac{a \tau+b}{c \tau+d}=\frac{a k \tau+b k}{\frac{c}{k} k \tau+d}$. It hence makes sense to associate to the motion $\gamma \in \Gamma_{o}(k)$ another motion $\hat{\gamma}=\left[\begin{array}{cc}a & b k \\ \frac{c}{k} & d\end{array}\right] \in \Gamma$, the full modular group ${ }^{8}$. Assume that $k$ is odd. Observe

${ }^{8}$ The map $\gamma \mapsto \hat{\gamma}$ is a group isomorphism of $\Gamma_{o}(k)$ onto $\Gamma^{o}(k)$. The definition of this last symbol can surely be left to the reader. It is clear that ^defines an automorphism of $\operatorname{PSL}(2, \mathbb{C})$ with the property that

$$
k \gamma(z)=\hat{\gamma}(k z),
$$

and

$$
\gamma^{\prime}(z)=\hat{\gamma}^{\prime}(k z),
$$

for all $\gamma \in \operatorname{PSL}(2, \mathbb{C})$ and all $z \in \mathbb{C} \cup\{\infty\}$. The automorphism ${ }^{\wedge}$ is conjugation by $\left[\begin{array}{cc}k^{\frac{1}{2}} & 0 \\ 0 & k^{-\frac{1}{2}}\end{array}\right]$. 
that for the characteristic $\chi=\left[\begin{array}{c}\frac{2 l+1}{k} \\ 1\end{array}\right]$ and $\tau \in \mathbb{H}^{2}$, we have

$$
\theta[\chi](0, k \gamma(\tau)) \gamma^{\prime}(\tau)^{\frac{1}{4}}=\theta[\chi](0, \hat{\gamma}(k \tau)) \hat{\gamma}^{\prime}(k \tau)^{\frac{1}{4}}=\kappa(\chi, \hat{\gamma}) \theta[\chi \hat{\gamma}](0, k \tau) \text {. }
$$

One easily sees that the characteristic $\chi \hat{\gamma}$ is equivalent to $\left[\begin{array}{c}\frac{2 l^{\prime}+1}{k} \\ 1\end{array}\right]$ for some integer $l^{\prime}$ with $0 \leq l^{\prime} \leq \frac{k-3}{2}$. Hence there is a permutation $\sigma=\sigma_{\gamma}$ of the integers in $\left[0, \frac{k-3}{2}\right]$ such that

$$
\gamma_{*} \varphi_{l}=\tilde{\kappa}\left(\chi_{l}, \hat{\gamma}\right) \varphi_{\sigma(l)}, l=0,1, \ldots, \frac{k-3}{2} .
$$

(The constant $\tilde{\kappa}$ is a $2 k$-th root of unity, and differs from $\kappa$ whenever $\chi_{\sigma(l)}-$ $\chi_{l} \hat{\gamma} \neq 0$ as characteristics.) It is a lengthy, but routine, calculation to show that each $\varphi_{l}$ is an eigenvector of $\gamma_{*}$ provided that $\gamma \in \Gamma(k)$ and that the eigenvalue is independent of $l$. For even $k$, one easily sees that the characteristic $\left[\begin{array}{c}\frac{2 l}{k} \\ 0\end{array}\right] \hat{\gamma}$ with $l \in \mathbb{Z}$ and $\gamma \in \Gamma_{o}(k)$ is equivalent to $\left[\begin{array}{c}\frac{2 l^{\prime}}{k} \\ 0\end{array}\right]$ for some $l^{\prime} \in \mathbb{Z}$. However $\varphi_{l}$ need NOT be an eigenvector for $\gamma_{*}$ even when $\gamma \in \Gamma(k)$. However, for such motions, for $l=0,1, \ldots, \frac{k}{2}, \gamma_{*}$ maps $\varphi_{l}$ into a constant nonzero multiple of either $\varphi_{l}$ or $\varphi_{\frac{k}{2}-l}$. Hence we have obtained

Lemma 4.4. (a) For odd $k$, the vector space $V(k)$ consists of e-automorphic functions for a factor of automorphy e for $\Gamma(k)$ of weight $\frac{1}{4}$.

(b) For even $k$, each $\varphi_{l}, l=0,1, \ldots, \frac{k}{2}$, is mapped by $\gamma_{*}, \gamma \in \Gamma(k)$, into a constant nonzero multiple of either $\varphi_{l}$ or $\varphi_{\frac{k}{2}-l}$.

Remark 2.If $k$ is an odd prime, and $\gamma_{1}$ and $\gamma_{2}$ belong to $\Gamma_{o}(k)$, then $\sigma_{\gamma_{1}}=$ $\sigma_{\gamma_{2}}$ if and only $a\left(\tilde{\gamma_{1}} \circ{\tilde{\gamma_{2}}}^{-1}\right) \equiv \pm 1 \bmod k$.

Remark 3. The above calculations have shown that $V_{o}(k)$ is $\Gamma_{o}(k)$-invariant. The Hilbert space $V_{o}(k)$ is not in general $\Gamma$-invariant (for example, from (4.5) we see that $A_{01} \neq 0$ for all $k>3$ ).

Remark 4. The theory for even $k$, leads to the appearence of new families of subgroups of $\Gamma$. Their study will be pursued elsewhere.

Lemmas 4.2 and 4.4 tell us that for odd $k$ we have a holomorphic map (see $\S 7$ for more details)

$$
\Phi: \overline{\mathbb{H}^{2} / \Gamma(k)} \rightarrow \mathbf{P} V(k) \cong \mathbf{P} \mathbb{C}^{\frac{k-3}{2}},
$$


and, as observed before, that we have a homomorphism

$$
\Theta: \text { Aut } \mathbb{H}^{2} / \Gamma(k) \cong \Gamma / \Gamma(k) \rightarrow \text { Aut } \mathbf{P} V(k) .
$$

The two maps are related; see (7.2). It is important to determine conditions under which $\Phi$ (hence also $\Theta$ ) is injective. See $\S 7$.

Assume that $k$ is odd. We have produced a homomorphism

$$
\sigma: \Gamma_{o}(k) \rightarrow \mathcal{S}_{\frac{k-1}{2}},
$$

where $\mathcal{S}_{N}$ is the permutation group on the $N$ nonnegative integers $\{0,1, \ldots$, $N-1\}$, whose kernel contains $G(k)$. For $\gamma \in \Gamma_{o}(k)$, the $\frac{k-1}{2} \times \frac{k-1}{2}$ matrix $\gamma_{*}$ has precisely one nonzero entry in each column. For the column $l=$ $0, \ldots, \frac{k-3}{2}$, the unique nonzero entry $\tilde{\kappa}\left(\chi_{l}, \tilde{\gamma}\right)$ is in the $\sigma_{\gamma}(l)$ row (rows are numbered 0 to $\frac{k-3}{2}$ ). Whereas $\sigma_{\gamma}$ depends only on $\gamma \in \Gamma_{o}(k) / G(k), \tilde{\kappa}$ depends on $\gamma \in \Gamma_{o}(k) / \Gamma(k)$. Assume for the remainder of this section that $k$ is an odd prime.

Lemma 4.5. For each odd prime $k, \Gamma_{o}(k) / G(k)$ is a cyclic group of order $\frac{k-1}{2}$.

Proof. We have the following inclusions of groups (with each group normal in the succeeding one):

$$
\Gamma(k) \triangleleft G(k) \triangleleft \Gamma_{o}(k) .
$$

By the first isomorphism theorem of group theory

$$
\Gamma_{o}(k) / G(k) \cong\left(\Gamma_{o}(k) / \Gamma(k)\right) /(G(k) / \Gamma(k)) .
$$

The groups $\Gamma_{o}(k) / \Gamma(k)$ and $G(k) / \Gamma(k)$ can be naturally identified with their images $\tilde{\Gamma}_{o}(k)$ and $\tilde{G}(k)$ in $\operatorname{SL}\left(2, \mathbb{Z}_{k}\right)$ under reduction $\bmod k$. The group $\tilde{G}(k)$ is cyclic of order $k$ with generator $B$ viewed as an element of $\operatorname{SL}\left(2, \mathbb{Z}_{k}\right)$. The elements of the group $\tilde{\Gamma}_{o}(k)$ are upper triangular. Therefore the problem is reduced to showing that the factor group of upper triangular elements in $\mathrm{SL}\left(2, \mathbb{Z}_{k}\right)$ modulo the upper triangular elements with diagonal elements 1 is a cyclic group. Let $\left[\begin{array}{ll}a & b \\ 0 & d\end{array}\right]$ be an arbitrary upper triangular matrix in $\mathrm{SL}\left(2, \mathbb{Z}_{k}\right)$. Then since

$$
\left[\begin{array}{ll}
a & b \\
0 & d
\end{array}\right]=\left[\begin{array}{ll}
a & 0 \\
0 & d
\end{array}\right]\left[\begin{array}{cc}
1 & b a^{-1} \\
0 & 1
\end{array}\right]
$$


it is clear that the coset decomposition of $\tilde{\Gamma}_{o}(k) / \tilde{G}(k)$ is of the form

$$
D_{1} \tilde{G}(k), \ldots, D_{\frac{k-1}{2}} \tilde{G}(k),
$$

with $D_{j}$ a diagonal matrix in $\mathrm{SL}\left(2, \mathbb{Z}_{k}\right)$. All that remains for us to do is show that the group of diagonal matrices in $\operatorname{SL}\left(2, \mathbb{Z}_{k}\right)$ is cyclic. For the diagonal terms of these matrices $a d \equiv 1$ modulo $k$. Hence each $a$ and $d$ is unit in the field $\mathbb{Z}_{k}$. The group of units in $\mathbb{Z}_{k}$ is cyclic of order $k-1$; so let $a$ be a generator. Hence $a^{k-1}=1$ in $\mathbb{Z}_{k}$, and no smaller integral power of $a$ will be the identity. It thus follows that $a^{\frac{k-1}{2}}=-1$. Similarly, $d^{\frac{k-1}{2}}=-1$. It follows that

$$
\left[\begin{array}{ll}
a & 0 \\
0 & d
\end{array}\right]
$$

has order $\frac{k-1}{2}$ in $\operatorname{PSL}\left(2, \mathbb{Z}_{k}\right)$.

Let $\gamma \in \Gamma_{o}(k)$ be a representative for the generator of the group $\Gamma_{o}(k) / G(k)$. This cyclic group of order $\frac{k-1}{2}$ acts transitively on the set of $\frac{k-1}{2}$ characteristics $\left\{\chi_{0}, \ldots, \chi_{\frac{k-3}{2}}\right\}$ (see [5]). It follows that $\sigma=\sigma_{\gamma}$ is cyclic permutation of the first $\frac{k-3}{2}$ nonnegative integers (in some order), and that hence that this permutation has no fixed points.

\section{Orders of automorphic forms at cusps.}

Our calculations of orders of automorphic forms are simplified by a number of simple observations. For each odd $k$ and $l=0,1, \ldots$ or $\frac{k-3}{2}$,

$$
\operatorname{ord}_{y+1} \varphi_{l}=\operatorname{ord}_{y} B_{*}\left(\varphi_{l}\right)=\operatorname{ord}_{y} \varphi_{l}
$$

and

$$
\operatorname{ord}_{-\frac{1}{y}} \varphi_{l}=\operatorname{ord}_{y} A_{*}\left(\varphi_{l}\right)=\operatorname{ord}_{y} \psi_{l},
$$

for all $y \in \mathbb{Q} \cup\{\infty\}$.

\subsection{Calculations via $\Gamma_{o}(k)$.}

As remarked in [5], it is a consequence of [20, Proposition 1.43] that the cusps $\infty$ and 0 project to the two punctures on $\mathbb{H}^{2} / \Gamma_{o}(k)$; thus every cusp in $\mathbb{Q} \cup\{\infty\}$ is $\Gamma_{o}(k)$-equivalent to either $\infty$ or 0 . 
Lemma 5.1. Let $l=0,1, \ldots$, or $\frac{k-3}{2}$. Then

(a) $\operatorname{ord}_{x} \varphi_{l}=\frac{1}{8}$, if the cusp $x \in \mathbb{Q} \cup\{\infty\}$ is $\Gamma_{o}(k)$-equivalent to zero.

(b) Let the cusp $x \in \mathbb{Q} \cup\{\infty\}$ be $\Gamma(k)$-equivalent to $\frac{j}{k}, j=1,2, \ldots, \frac{k-1}{2}$ (this is a complete list of representatives for the $\Gamma(k)$-equivalence classes of cusps that are $\Gamma_{o}(k)$-equivalent to infinity). Choose $a \gamma \in \Gamma_{o}(k)$ such that $\gamma(\infty)=x$. Then

$$
\operatorname{ord}_{x} \varphi_{l}=\frac{\left(2 \sigma_{\gamma}(l)+1\right)^{2}}{8}
$$

Proof. For $x=0$, part (a) is a consequence of (4.4). For any $\gamma \in \Gamma$, and every $y \in \mathbb{H}^{2} \cup \mathbb{Q} \cup\{\infty\}$, we have

$$
\operatorname{ord}_{\gamma(y)} \varphi_{l}=\operatorname{ord}_{y}\left(\gamma_{*} \varphi_{l}\right) .
$$

If we take $y=0$ and $\gamma \in \Gamma_{o}(k)$, then using (4.8) we obtain

$$
\operatorname{ord}_{\gamma(0)} \varphi_{l}=\operatorname{ord}_{0} \varphi_{\sigma_{\gamma}(l)}
$$

which completes the proof of part (a). We next take $y=\infty$ and $\gamma \in \Gamma_{o}(k)$. Using (4.8), once again, and (4.1), we obtain

$$
\operatorname{ord}_{\gamma(\infty)} \varphi_{l}=\operatorname{ord}_{\infty} \varphi_{\sigma_{\gamma}(l)}=\frac{\left(2 \sigma_{\gamma}(l)+1\right)^{2}}{8} .
$$

Definition 1. We will call a puncture on $\mathbb{H}^{2} / \Gamma(k)$ distinguished if it is the image under $P$ of a cusp that is $\Gamma_{o}(k)$-equivalent to $\infty$. This definition makes sense for arbitrary $k$. For $k$ an odd prime, these are the punctures $\left\{P_{\frac{j}{k}}, j=1, \ldots, \frac{k-1}{2}\right\}$.

Remark 5. The $\frac{k^{2}-1}{2}$ punctures on $\mathbb{H}^{2} / \Gamma(k)$ split up into $\frac{k+1}{2}$ disjoint sets; one of these sets consists of the $\frac{k-1}{2}$ distinguished punctures, each fixed by $\tilde{B}$, and each of the other $\frac{k-1}{2}$ sets consists of $k$ punctures, cyclically permuted by $\langle\tilde{B}\rangle$. The $k \frac{k-1}{2}$ nondistinguished punctures are $\Gamma_{o}(k) / \Gamma(k)$-equivalent to $P_{0}$.

Remark 6. The above lemma (except for the parenthetical remark) also holds for odd composite $k$. However, in the composite case there are cusps that are $\Gamma_{o}(k)$-equivalent to neither 0 nor $\infty$. To compute the order of a modified theta constant at one of these cusps, we must use the methods discussed in next subsection. 
Remark 7. We saw in [5] that if we identify the puncture $P_{\infty}$ with the class of the characteristic $\chi_{o}$, then for each $\gamma \in \Gamma$, the puncture $P_{\gamma^{-1}(\infty)}$ is naturally identified with the class of the characteristic $\chi_{o} \gamma$. Similarly, if we identify the puncture $P_{\infty}$ with the class of the characteristic $\chi_{o} A$ or $\chi_{0}$, the puncture $P_{\gamma^{-1}}(\infty)$ is naturally identified with the class of the characteristic $\chi_{0} \gamma$. Further, the distinguished punctures can be listed as $P_{\gamma_{j}^{-1}(\infty)}$, with $\left\{\gamma_{1}, \ldots, \gamma_{\frac{k-1}{2}}\right\}$ a complete set of representatives for the factor group $G(k) \backslash \Gamma_{o}(k)$. The corresponding list of characteristics is then $\mathcal{T}_{1} A$ (the characteristics $\left\{\chi_{0}, \ldots, \chi_{\frac{k-3}{2}}\right\}$ ).

Lemma 5.2. Let $l$ be an integer with $0 \leq l \leq \frac{k-3}{2}$. As $x$ in the last lemma runs over representatives of the distinct $\Gamma(k)$-equivalence classes of cusps $\Gamma_{o}(k)$-equivalent to $\infty$, the corresponding integers $\sigma_{\gamma}(l)$ run over the nonnegative integers $\left\{0,1, \ldots, \frac{k-3}{2}\right\}$.

Proof. We use the fact that $\Gamma_{o}(k) / G(k)$ is cyclic of order $\frac{k-1}{2}$ and in oneto-one correspondence with the tower $\mathcal{T}_{1}$ in $X_{o}(k)$, the basis for $V(k)$ given by the functions $\left\{\varphi_{0}, \ldots, \varphi_{\frac{k-3}{2}}\right\}$, and the distinguished punctures $\left\{P_{\infty}=\right.$ $\left.x_{1}, \ldots, x_{\frac{k-1}{2}}\right\}$ on $\mathbb{H}^{2} / \Gamma(k)$ determined by the cusps $\Gamma_{o}(k)$-equivalent to $\infty$. Choose a generator $\gamma$ of $\Gamma_{o}(k) / G(k)$. We reorder the punctures $\left\{x_{2}, \ldots\right.$, $\left.x_{\frac{k-1}{2}}\right\}$, if needed, so that $\gamma\left(x_{i}\right)=x_{i+1}$ (we are using two identifications in this last equation: punctures are identified with cusps that project onto them, and addition is interpreted modulo $\frac{k-1}{2}$ ). Then we can rewrite (5.3) as

$$
\operatorname{ord}_{x_{j}} \varphi_{l}=\frac{\left(2 \sigma^{j-1}(l)+1\right)^{2}}{8},
$$

where $\sigma=\sigma_{\gamma}$. Since $\langle\tilde{\gamma}>$ acts cyclically on the distinguished punctures $\left\{x_{1}, \ldots, x_{\frac{k-1}{2}}\right\}$ (or equivalently on the classes of the characteristics $\left\{\chi_{0}, \ldots, \chi_{\frac{k-3}{2}}\right\}$ in some order), for each $l$, the powers of $\sigma$ evaluated at $l$ define an element of $\mathcal{S}_{\frac{k-1}{2}}$.

We record the following useful

Corollary 5.3. Let $\gamma \in \Gamma_{o}(k)$. If $\sigma_{\gamma}(l)=l$ for some integer $l$ with $0 \leq l \leq$ $\frac{k-3}{2}$, then $\gamma \in G(k)$.

Corollary 5.4. For each integer $l$ with $0 \leq l \leq \frac{k-3}{2}$, the orders of $\varphi_{l}$ at the $\frac{k-1}{2}$ distinguished punctures are distinct. 
Remark 8. The last two lemmas imply that

$$
\operatorname{deg}\left(\varphi_{l}\right)=\frac{1}{48} k\left(k^{2}-1\right)=\frac{1}{4}(2 p(k)-2+n(k)),
$$

as expected. It is also of interest to note that the generic element of $V(k)$ has $\frac{\left(k^{2}-1\right)(k-3)}{48}$ simple $\Gamma(k)$-inequivalent zeros on $\mathbb{H}^{2}$.

\subsection{The general case.}

In order to develop a general alternate method for computing $\operatorname{ord}_{x} \varphi_{l}$ at an arbitrary cusp $x$ for $\Gamma(k)$, we must study in. some detail the invariance of modified theta constants under $\Gamma$. We need an analogue of (3.4) for elements $\tilde{\gamma}$ with $\gamma \in \operatorname{PSL}(2, \mathbb{Z})$.

If $C \in \Gamma$, then we know that for every integer $l$ with $0 \leq l \leq \frac{k-3}{2}$, we have

$$
\operatorname{ord}_{C(\infty)} \varphi_{l}=\operatorname{ord}_{\infty}\left(C_{*}^{\prime} \varphi_{l}\right)
$$

Now for $\tau \in \mathbb{H}^{2}$,

$$
\left(C_{*} \varphi_{l}\right)(\tau)=\theta\left[\begin{array}{c}
\frac{2 l+1}{k} \\
1
\end{array}\right](0, k C(\tau)) C^{\prime}(\tau)^{\frac{1}{4}}=\theta\left[\begin{array}{c}
\frac{2 l+1}{k} \\
1
\end{array}\right](0, \hat{C}(k \tau)) \hat{C}^{\prime}(k \tau)^{\frac{1}{4}} .
$$

If $C \in \Gamma_{o}(k)$, we can continue the last equation and obtain

$$
\left(C_{*} \varphi_{l}\right)(\tau)=\kappa\left(\left[\begin{array}{c}
\frac{2 l+1}{k} \\
1
\end{array}\right], \hat{C}\right) \theta\left[\left[\begin{array}{c}
\frac{2 l+1}{k} \\
1
\end{array}\right] \hat{C}\right](0, k \tau) .
$$

From which it follows, as seen before, that

$$
\operatorname{ord}_{C(\infty)} \varphi_{l}=\operatorname{ord}_{\infty} \varphi_{\sigma_{C}(l)}
$$

However, if $C \in \Gamma-\Gamma_{o}(k)$, we need an alternate approach. We have seen that ${ }^{9}$

$$
B_{*} \varphi_{l}=c \varphi_{l} \text { and } A_{*} \varphi_{l}=c \psi_{l} .
$$

An arbitrary element $C \in \Gamma$ is a word in the generators $B$ and $A$. Hence of the form

$$
B^{n_{1}} \circ A \circ B^{n_{2}} \circ A \circ B^{n_{3}} \ldots \circ A \circ B^{n_{r}},
$$

\footnotetext{
${ }^{9} \mathrm{In}$ each of the next two displayed equations $c$ is a nonzero constant, not necessarily the same in the two equations, that can easily be computed.
} 
for some integer $r>1$, where the indices

$$
n_{1}, n_{2}, \ldots, n_{r}
$$

are integers with all but the first and last nonzero. This formula, can be used to determine the action of an arbitrary unimodular matrix on the modified theta constants.

\section{The field of meromorphic functions on $\overline{\mathbb{H}^{2} / \Gamma(k)}$.}

\subsection{Functions of small degree.}

As a consequence of Lemmas 4.4 and 5.1, for odd $k$, a ratio of any two linearly independent functions in $V(k)$ defines a meromorphic function on $\overline{\mathbb{H}^{2} / \Gamma(k)}$. In particular, if $l$ and $l^{\prime}$ are unequal nonnegative integers $\leq \frac{k-3}{2}$, then $\frac{\varphi_{l}}{\varphi_{l^{\prime}}}$ projects to such a function. We are interested in producing functions of low degree.

Let us assume that $k$ is an odd prime. The divisor of the projection of $\frac{\varphi_{l}}{\varphi_{l^{\prime}}}$ to $\overline{\mathbb{H}^{2} / \Gamma(k)}$ is supported at the $\frac{k-1}{2}$ punctures that are $\Gamma_{o}(k)$-equivalent to $\infty$. It can have either zeros or poles at at most half of these points. Without loss of generality we assume that the function has fewer (or equal number of) zeros than poles. We estimate the degree of such a function by counting the number of zeros (with multiplicities) that it has. An upper bound for the order of a zero of $\frac{\varphi_{l}}{\varphi_{l^{\prime}}}$ at a point is obtained by maximizing the order of the zero of $\varphi_{l}$ and minimizing the one of $\varphi_{l^{\prime}}$. Using the fact the the orders of the zeros of one of these automorphic forms at the $\frac{k-1}{2}$ distinguished punctures are permutations of the rational numbers

$$
\frac{1^{2}}{8}, \frac{3^{2}}{8}, \ldots, \frac{(k-2)^{2}}{8},
$$

we conclude ${ }^{10}$ that we can produce such a function of degree at most

$$
\frac{1}{64}(k-1)^{3}, \text { if } k \equiv 1 \bmod 4 \text { and } \frac{1}{128}(2 k-5)\left(k^{2}-1\right), \text { if } k \equiv 3 \bmod 4 .
$$

\footnotetext{
${ }^{10}$ We use the following well known formula. For the positive odd integer $n$, we have

$$
\sum_{i=0}^{\frac{n-1}{2}}(2 i+1)^{2}=\frac{1}{6} n(n+1)(n+2) .
$$
}


We have shown that for odd primes $k$, the compact surface $\overline{\mathbb{H}^{2} / \Gamma(k)}$ carries a function of degree $O\left(\frac{k^{3}}{64}\right), k \rightarrow \infty$ which is smaller than its genus: $O\left(\frac{k^{3}}{24}\right)$, $k \rightarrow \infty$, and an improvement on the result for generic surfaces $\left(O\left(\frac{k^{3}}{48}\right)\right.$, $k \rightarrow \infty$; see, for example, [9, pg. 261]). The method we have used can probably be modified to guarantee the existence of functions of lower degree.

The Hilbert space $V(k)$ allows us to study a number of interesting divisors ${ }^{11}$ on $\overline{\mathbb{H}^{2} / \Gamma(k)}$.

Proposition 6.1. For each integer $l, 0 \leq l \leq \frac{k-3}{2}$, let

$$
D_{l}=\prod_{j=1}^{\frac{k-1}{2}} P_{\frac{j}{k}}^{\operatorname{ord}_{\frac{j}{k}} \varphi_{l}-\frac{1}{8}} .
$$

Then

$$
i\left(D_{l}\right) \geq \frac{(k-1)(k-3)(k-5)}{48} .
$$

Proof. We first observe, for future use, that

$$
\operatorname{deg} D_{l}=\frac{1}{48}\left(k^{2}-1\right)(k-3)<p,
$$

for $k \geq 11$. Since for every $\varphi \in V(k)$, the projection of $\frac{\varphi}{\varphi_{l}}$ to $\overline{\mathbb{H}^{2} / \Gamma(k)}$ belongs to $L\left(\frac{1}{D_{l}}\right)$, we conclude that

$$
r\left(\frac{1}{D_{l}}\right) \geq \frac{k-1}{2}
$$

The inequality on the index of specialty follows from the Riemann-Roch theorem.

Corollary 6.2. The class of the divisor $D_{l}$ is independent of $l$.

Proof. For $l$ and $l^{\prime}$ as above $\frac{\Phi_{l}}{\Phi_{l^{\prime}}}$ is a meromorphic function on $\overline{\mathbb{H}^{2} / \Gamma(k)}$ with divisor $\frac{D_{l}}{D_{l^{\prime}}}$.

\footnotetext{
${ }^{11}$ We use, throughout this paper, standard (multiplicative) notation ([6, Chapter III]) for divisors and the indices appearing in the Riemann-Roch theorem.
} 
Remark 9. For certain low primes $k$, we will reprove the above proposition in the sequel. Note that for $k=7$, the divisors $D_{l}$ have degree 4 , and are hence canonical as a consequence of the proposition. The proposition implies trivially the existence of a meromorphic function of degree at most $\frac{1}{48}\left(k^{2}-1\right)(k-3)$ on $\overline{\mathbb{H}^{2} / \Gamma(k)}$.

\section{2. $G(k)$-invariant functions.}

The material of this subsection is valid for odd integers $k \geq 3$. Let $\alpha_{i} \in \mathbb{Z}$ for $i=0,1, \ldots, \frac{k-3}{2}$, be chosen so that

$$
\sum_{i=0}^{\frac{k-3}{2}} \alpha_{i}=0 \text { and } k \mid \sum_{i=0}^{\frac{k-3}{2}}\left(i+i^{2}\right) \alpha_{i} .
$$

Then

$$
\Pi_{i=0}^{\frac{k-3}{2}} \varphi_{i}^{\alpha_{i}}
$$

is a $G(k)$-invariant meromorphic function on $\mathbb{H}^{2}$. It hence makes sense to call $\left(\alpha_{0}, \ldots, \alpha_{\frac{k-3}{2}}\right)$ an admissible $\frac{k-1}{2}$-tuple if it satisfies the above conditions.

For example, for $k=7$, we can choose as the triple of integers

$$
(3,-1,-2) \text {, or }(1,2,-3) \text {, or }(-1,-2,3) \text {. }
$$

For $k=11$, we can choose the 5 integers as

$$
(2,-1,0,0,-1) \text { or }(-1,0,2,-1,0),
$$

and for $k=13$ the 6 integers as

$$
(-1,1,-1,0,0,1) \text {. }
$$

Assume that the prime $k \geq 7$. Given any collection of $\frac{k-1}{2}-2$ integers, $\alpha_{2}, \alpha_{3}, \ldots, \alpha_{\frac{k-3}{2}}$, it can always be completed to an admissible $\frac{k-1}{2}$-tuple by setting

$$
\alpha_{1}=k r-\sum_{i=2}^{\frac{k-3}{2}} \frac{i+i^{2}}{2} \alpha_{i} \text { and } \alpha_{0}=-\sum_{i=1}^{\frac{k-3}{2}} \alpha_{i},
$$

where $r \in \mathbb{Z}$ is arbitrary. In particular,

$$
\left(\frac{k-3}{2},-\frac{k-3}{2}, 0, \ldots, 0,-1,1\right),(-3,3,0, \ldots, 0,-1,1)
$$




$$
(2,-3,1,0, \ldots, 0) \text {, and }(-k+2, k-3,1,0, \ldots, 0)
$$

are always a nontrivial admissible $\frac{k-1}{2}$-tuples.

\subsection{Generators for the function field.}

Let us restrict our attention to primes $k \geq 7$. It is convenient to work with the functions

$$
f_{1}=\frac{\varphi_{1}}{\varphi_{0}}, \text { and } f_{2}=\frac{\varphi_{2}}{\varphi_{1}} \text {. }
$$

The divisors of the functions $F_{1}$ and $F_{2}$ are supported at the $\frac{k-1}{2}$ distinguished punctures $\left\{P_{\frac{j}{k}} ; j \in \mathbb{Z}, 1 \leq j \leq \frac{k-1}{2}\right\}$. For each such $j$ we can choose integers $b=b(j)$ and $d=d(j)$ such that

$$
j d-b k=1 .
$$

Then the Möbius transformation

$$
\gamma_{j}=\left[\begin{array}{cc}
j & b \\
k & d
\end{array}\right] \in \Gamma_{o}(k)
$$

and $\gamma_{j}(\infty)=\frac{j}{k}$. We have already seen that (the definition of $m(\cdot)$ is given in [5])

$$
\left(F_{1}\right)=\prod_{j=1}^{\frac{k-1}{2}} P_{\frac{j}{k}}^{\frac{m\left(\chi_{1} \tilde{\gamma_{j}}\right)^{2}-m\left(\chi_{0} \tilde{\gamma}_{j}\right)^{2}}{8}} \text { and }\left(F_{2}\right)=\prod_{j=1}^{\frac{k-1}{2}} P_{\frac{j}{k}}^{\frac{m\left(\chi_{2} \tilde{\gamma}_{j}\right)^{2}-m\left(\chi_{1} \tilde{\gamma}_{j}\right)^{2}}{8}} .
$$

An examination of cases shows that there are only two possibilities for the jump in the function $m$ on characteristics; for example:

$$
m\left(\chi_{1} \tilde{\gamma}_{j}\right)=m\left(\chi_{0} \tilde{\gamma}_{j}\right)+2 j \text { if } 1 \leq j \leq \frac{k-m\left(\chi_{0} \tilde{\gamma}_{j}\right)}{2}
$$

and

$$
m\left(\chi_{1} \tilde{\gamma_{j}}\right)=2(k-j)-m\left(\chi_{0} \tilde{\gamma_{j}}\right) \text { if } \frac{k-m\left(\chi_{0} \tilde{\gamma_{j}}\right)}{2}<j \leq \frac{k-1}{2} .
$$

We are dealing with characteristics $\chi$ of the form $\left[\begin{array}{c}\frac{m}{k} \\ 1\end{array}\right]$, with $m \in \mathbb{Z}, m$ odd, and $1 \leq m \leq k-2$. Thus the equivalence class of $\chi$ is completely determined by $m(\chi)$. We conclude that each $F_{i}$ has either a pole or a zero 
at each puncture $P_{\frac{j}{k}}$. Let $Q_{1}, \ldots, Q_{r}$ be the complete list of distinct zeros of $F_{1}$. Then in some local coordinate $t$ vanishing at $Q_{i}$, we can write

$$
z=F_{1}(t)=t^{\alpha}
$$

for some $\alpha \in \mathbb{Z}, \alpha>0$, and

$$
w=F_{2}(z)=\sum_{j=\beta}^{\infty} c_{j} z^{\frac{j}{\alpha}},
$$

where $\beta \in \mathbb{Z}, \beta \neq 0$, and the $c$ 's are the appropriate Laurent series coefficients. Of course,

$$
\alpha=\operatorname{ord}_{P_{\infty}} F_{1} \text { and } \beta=\operatorname{ord}_{P_{\infty}} F_{2} .
$$

We study (see $\S 2$ ) the map $\varphi: \overline{\mathbb{H}^{2} / \Gamma(k)} \rightarrow \mathfrak{M}^{*}$ defined by the functions $z=F_{1}$ and $w=F_{2}$. Since

$$
\operatorname{ord}_{P_{\infty}} F_{1}=1 \text { and } \operatorname{ord}_{P_{\infty}} F_{2}=2,
$$

$\varphi\left(P_{\infty}\right)$ is the Puiseaux series

$$
w(z)=c z^{2}+\ldots .
$$

If $\varphi(P)=\varphi\left(P_{\infty}\right)$ for some $P \in \overline{\mathbb{H}^{2} / \Gamma(k)}$, then we must have

$$
\operatorname{ord}_{P} F_{1}=\alpha \text { and } \operatorname{ord}_{P} F_{2}=2 \alpha,
$$

for some positive integer $\alpha$. Thus $P=P_{\frac{j}{k}}$ for some integer $j$ with $1 \leq j \leq$ $\frac{k-1}{2}$. If $j \neq 1$, then we must have that

$$
\alpha=\frac{m_{2}^{2}-m_{1}^{2}}{8} \text { and } 2 \alpha=\frac{m_{3}^{2}-m_{2}^{2}}{8},
$$

where $m_{1}, m_{2}$, and $m_{3}$ are odd integers with

$$
1<m_{1}<m_{2}<m_{3}<k
$$

which leads to the diophantine equation

$$
m_{3}^{2}-3 m_{2}^{2}+2 m_{1}^{2}=0 .
$$

Under special circumstances it might be possible to determine solutions of these equations and decide when $\varphi$ is injective. It is easier to use more 
terms of the respective Taylor series expansions. In terms of the natural coordinate $\zeta$ in a neighborhood of $P_{\infty}$, we have

$$
z=\frac{\Phi_{1}(\zeta)}{\Phi_{0}(\zeta)}=F_{1}(\zeta)=\exp \left(\frac{\pi \imath}{k}\right) \zeta\left(1-\zeta^{k \frac{k-3}{2}}+\ldots\right)
$$

and

$$
w=\frac{\Phi_{2}(\zeta)}{\Phi_{1}(\zeta)}=F_{2}(\zeta)=\exp \left(\frac{\pi \imath}{k}\right) \zeta^{2}\left(1-\zeta^{k \frac{k-5}{2}}+\ldots\right) .
$$

Hence for $\varphi\left(P_{\frac{1}{k}}\right)$ we have

$$
w=w(z)=c_{0} z^{2}\left(1+c_{1} z+\ldots\right) .
$$

In the above and subsequent equations in this subsection, $c_{i}$, with $i \in \mathbb{Z}$, is a nonzero constant (that can easily be computed). The last three displayed equations show that $b_{\varphi}\left(P_{\infty}\right)=0^{12}$. Thus to show that $\operatorname{deg} \varphi=1$, it suffices $^{13}$ to prove that $\varphi(P) \neq \varphi\left(P_{\frac{1}{k}}\right)$ for all $P \in \overline{\mathbb{H}^{2} / \Gamma(k)}, P \neq P_{\frac{1}{k}}$. If $\varphi(P)=\varphi\left(P_{\frac{1}{k}}\right)$ for some $P \in \overline{\mathbb{H}^{2} / \Gamma(k)}$, then it must be the case as above that $P=P_{\frac{j}{k}}$ for some integer $j$ with $1 \leq j \leq \frac{k-1}{2}$. We need to obtain the Taylor series for $z$ and $w$ in terms of a good local coordinate vanishing at $P_{\frac{j}{k}}$. The functions $z$ and $w$ are the projections to $\overline{\mathbb{H} \mathbb{I}^{2} / \Gamma(k)}$ of the $\Gamma(k)$-invariant functions $\frac{\varphi_{1}}{\varphi_{0}}$ and $\frac{\varphi_{2}}{\varphi_{1}}$ on $\mathbb{H}^{2} \cup \mathbb{Q} \cup\{\infty\}$. Choose $\gamma \in \Gamma_{o}(k)$ such that

$$
\gamma(\infty)=\frac{j}{k}
$$

Thus we want to study

$$
\frac{\varphi_{1} \circ \gamma}{\varphi_{0} \circ \gamma} \text { and } \frac{\varphi_{2} \circ \gamma}{\varphi_{1} \circ \gamma}
$$

in a neighborhood of $\imath \infty$. From (4.8) it follows that

$$
\frac{\varphi_{1} \circ \gamma}{\varphi_{0} \circ \gamma}=\frac{\tilde{\kappa}\left(\chi_{1}, \tilde{\gamma}\right)}{\tilde{\kappa}\left(\chi_{0}, \tilde{\gamma}\right)} \frac{\varphi_{\sigma(1)}}{\varphi_{\sigma(0)}} \text { and } \frac{\varphi_{2} \circ \gamma}{\varphi_{1} \circ \gamma}=\frac{\tilde{\kappa}\left(\chi_{2}, \tilde{\gamma}\right)}{\tilde{\kappa}\left(\chi_{1}, \tilde{\gamma}\right)} \frac{\varphi_{\sigma(2)}}{\varphi_{\sigma(1)}}
$$

with $\sigma=\sigma_{\gamma}$. Thus, for these purposes,

$$
z=\alpha \frac{\Phi_{l}}{\Phi_{l^{\prime}}} \text { and } w=\beta \frac{\Phi_{m}}{\Phi_{l}}
$$

${ }^{12}$ The function $z$ is a perfectly good local coordinate at both $P_{\infty} \in \overline{\mathbb{H}^{2} / \Gamma(k)}$ and at $\varphi\left(P_{\infty}\right) \in \mathfrak{M}^{*}$. In terms of these coordinates $\varphi$ is the identity map.

${ }^{13}$ We use the symbol $P$ to denote at times a point on $\overline{\mathbb{H}^{2} / \Gamma(k)}$ and the natural projection $\mathbb{H}^{2} \cup \mathbb{Q} \cup\{\infty\} \rightarrow \overline{\mathbb{H}^{2} / \Gamma(k)}$. This identification should cause no confusion to the reader. 
for distinct nonnegative integers $l, l^{\prime}$, and $m$ each at most $\frac{k-3}{2}$ and constants $\alpha$ and $\beta$ of absolute value 1. Further, if $j \neq 1$, then $\sigma(0)>0$ (because $\sigma$ acts without fixed points) and

$$
m>l>l^{\prime}>0
$$

(the first two inequalities are consequences of the fact that both $z$ and $w$ vanish at $\left.P_{\frac{j}{k}}\right)$. It follows that for $\varphi(P)$ we have

$$
w=w(z)=c_{2} z^{\frac{(m+l+1)(m-l)}{\left(l+l^{\prime}+1\right)\left(l-l^{\prime}\right)}}\left(1+c_{3} z^{\frac{2}{\left(l+l^{\prime}+1\right)\left(l-l^{\prime}\right)}}+\ldots\right) .
$$

Hence

$$
2=\frac{(m+l+1)(m-l)}{\left(l+l^{\prime}+1\right)\left(l-l^{\prime}\right)} \text { and } 1=\frac{2}{\left(l+l^{\prime}+1\right)\left(l-l^{\prime}\right)} .
$$

We have arrived at a contradiction since

$$
\left(l+l^{\prime}+1\right)\left(l-l^{\prime}\right) \geq 4
$$

and hence obtained

Theorem 6.3. For each prime $k \geq 7$, the functions $F_{1}$ and $F_{2}$ generate the function field $\mathcal{K}\left(\overline{\mathbb{H}^{2} / \Gamma(k)}\right)$.

The same methods establish

Theorem 6.4. For each prime $k \geq 7$, the functions $J$ and $F_{1}$ generate the function field $\mathcal{K}\left(\overline{\mathbb{H}^{2} / \Gamma(k)}\right)$.

Problem 1. Find the algebraic relation satisfied by $F_{1}$ and $F_{2}$. For $k=7$ we solve this problem in $\S 10$. The form of the algebraic equation satisfied by $J$ and $F_{1}$ is given in $\S 14$.

\section{Projective representations.}

A coordinate vector in the finite dimensional projective space $\mathbf{P} \mathbb{C}^{\frac{k-3}{2}}$ is the equivalence class of a vector in $\mathbb{C}^{\frac{k-1}{2}}$ with precisely one nonzero coordinate. For $l=0,1, \ldots, \frac{k-5}{2}$,

$$
f_{l}=\frac{\varphi_{l}}{\varphi_{\frac{k-3}{2}}}
$$

is a meromorphic function on the compact surface $\overline{\mathbb{H}^{2} / \Gamma(k)}$ whose divisor is supported at the distinguished punctures of $\mathbb{H}^{2} / \Gamma(k)$. 
Lemma 7.1. Let $x \in \mathbb{Q} \cup\{\infty\}$. If

$$
\operatorname{ord}_{x} \varphi_{l}>\operatorname{ord}_{x} \varphi_{0}, \text { for } l=1, \ldots, \frac{k-3}{2}
$$

then $P_{x}=P_{\infty}$.

Proof. For each fixed $l$ as above, the function $\frac{\Phi_{l}}{\Phi_{0}}$ vanishes at $P_{x}$. Hence $P_{x}$ is a distinguished puncture. Thus $x=\gamma(\infty)$ for some $\gamma \in \Gamma_{o}(k)$. Now for arbitrary $l$ as above, we have

$$
\operatorname{ord}_{\gamma(\infty)} \varphi_{l}=\operatorname{ord}_{\infty} \gamma_{*}\left(\varphi_{l}\right)=\operatorname{ord}_{\infty} \varphi_{\sigma_{\gamma}(l)} .
$$

We conclude that $\sigma_{\gamma}(l)>0$ for all $l>0$. Thus $\sigma_{\gamma}(0)=0$, and hence $\gamma \in G(k)$. It now follows that $x$ and $\infty$ project to the same puncture on $\mathbb{H}^{2} / \Gamma(k)$.

Proposition 7.2. For each odd integer $k \geq 5$, the map

$$
\tau \mapsto\left(f_{0}(\tau), f_{1}(\tau), \ldots, f_{\frac{k-5}{2}}(\tau), 1\right)
$$

from $\mathbb{H}^{2} \cup \mathbb{Q} \cup\{\infty\}$ to $\mathbb{C}^{\frac{k-1}{2}}$, or equivalently the map

$$
\Phi: \tau \mapsto\left(\varphi_{0}(\tau), \varphi_{1}(\tau), \ldots, \varphi_{\frac{k-5}{2}}(\tau), \varphi_{\frac{k-3}{2}}(\tau)\right)
$$

from $\mathbb{H}^{2} \cup \mathbb{Q} \cup\{\infty\}$ to $\mathbb{C}^{\frac{k-1}{2}}$, defines a holomorphic mapping (also to be called $\Phi)$ from $\overline{\mathbb{H}^{2} / \Gamma(k)}$ into $\mathbf{P} \mathbb{C}^{\frac{k-3}{2}}$. If $k$ is prime, the distinguished punctures on the surface $\mathbb{H}^{2} / \Gamma(k)$ are sent (injectively) onto the coordinate vectors in projective space $\mathbf{P} \mathbb{C}^{\frac{k-3}{2}}$. Further, in this case, the map $\Phi$ restricted to the punctures is injective, and of maximal rank at each distinguished puncture.

Proof. Only the last three statements need verification.

We need to study the map $\Phi$ in some detail. It is convenient to view $\Phi$ as a holomorphic map from $\overline{\mathbb{H}^{2} / \Gamma(k)}$ into $\mathbf{P} V(k)^{*}$, the space of continuous linear functionals on (lines in) $\mathbf{P} V(k)$. In this setting $\Phi(P)$ for $P \in \overline{\mathbb{H}^{2} / \Gamma(k)}$ is the projective equivalence class of the linear functional ${ }^{14}$

$$
L_{P}: \varphi \mapsto \varphi(P), \varphi \in V(k) .
$$

\footnotetext{
${ }^{14}$ In the next and many subsequent formulae we identify points in on the surface $\overline{\mathbb{H}^{2} / \Gamma(k)}$ with their preimages under $P$ in $\mathbb{H}^{2} \cup \mathbb{Q} \cup\{\alpha\}$, and $\Gamma(k)$-invariant functions on $\mathbb{H}^{2} \cup \mathbb{Q} \cup\{\infty\}$ with their projections to $\left(\mathbb{H}^{2} \cup \mathbb{Q} \cup\{\infty\}\right) / \Gamma(k)$.
} 
In this setting, (7.1) identifies $L_{P_{\tau}}$ with the matrix representation of this linear functional with respect to the basis $\left\{\varphi_{0}, \ldots, \varphi_{\frac{k-3}{2}}\right\}$. If, as can happen (for example, at $\left.P=P_{\infty}\right), \varphi(P)=0$ for all $\varphi \in V(k)^{2}$, then we let $l$ be the minimum of the orders of vanishing at $P$ of the nonzero elements in $V(k)$ and interpret $L_{P}$ as evaluation at 0 of the functions in $\frac{V(k)}{\zeta^{l}}$, where $\zeta$ is a local coordinate vanishing at $P$. A similar interpretation applies to the map $F$ in neighborhoods of points where the meromorphic functions have poles (or vanish simultaneously).

An element $\gamma \in \Gamma / \Gamma(k)$ acts as an automorphism $\gamma$ of the Riemann surface $\overline{\mathbb{H}^{2} / \Gamma(k)}$, a projective linear automorphism $\gamma^{*}$ of $\mathbf{P} V(k)$, and a projective linear automorphism $\gamma^{*}$ (where $\gamma^{*}(L)=L\left(\gamma_{*}\right)$ for $\left.L \in \mathbf{P} V(k)^{*}\right)$ of the projective space $\mathbf{P} V(k)^{*}$ such that

$$
\Phi \circ \gamma=\gamma^{*} \circ \Phi
$$

We use the elements of $\Gamma$, to compute the images in $\mathbf{P} V(k)^{*}$ of the punctures on $\mathbb{H}^{2} / \Gamma(k)$ under the map $\Phi$. We observe that

$$
\Phi(\infty)=(1,0, \ldots, 0) .
$$

If the cusp $x \in \mathbb{Q}$ is $\Gamma_{o}(k)$-equivalent to $\infty$, then we choose $\gamma \in \Gamma_{o}(k)$ with $\gamma(\infty)=x$. Then

$$
\begin{aligned}
\Phi(x)=L_{x} & =L_{\gamma(\infty)}= \\
& =\gamma^{*}\left(L_{\infty}\right)=\left(\tilde{\kappa}\left(\chi_{0}, \tilde{\gamma}\right) \varphi_{\sigma(0)}(\infty), \ldots, \tilde{\kappa}\left(\chi_{\frac{k-3}{2}}, \tilde{\gamma}\right) \varphi_{\sigma\left(\frac{k-3}{2}\right)}(\infty)\right),
\end{aligned}
$$

where $\sigma=\sigma_{\gamma}$. It follows that $\Phi(x)$ is a coordinate vector with the unique nonzero component in the $\sigma^{-1}(0)+1$ slot. Taking $\gamma$ to be a generator for the cyclic group $\Gamma_{o}(k) / G(k)$, we obtain a cyclic permutation $\sigma$ which implies immediately the injectivity of $\Phi$ restricted to the distinguished punctures. For the general case, assume that $\Phi(x)=\Phi(y)$ with $x$ and $y$ in $\mathbb{Q} \cup\{\infty\}$. Write $x=\gamma(\infty)$ with $\gamma \in \Gamma$. Then with $z=\gamma^{-1}(y)$, we have

$$
\gamma^{*}(\Phi(\infty))=\Phi(\gamma(\infty))=\Phi(\gamma(z))=\gamma^{*}(\Phi(z)) .
$$

Since $\gamma^{*}$ is injective, $\Phi(\infty)=\Phi(z)$. We conclude from the previous lemma that $P_{\infty}=P_{z}$. Hence $\infty$ is $\Gamma(k)$-equivalent to $\gamma^{-1}(y)$; that is, $x=\gamma(\infty)$ is $\Gamma(k)$-equivalent to $y$. Finally in a neighborhood of $P_{\infty}$ the map $\Phi$ is given in inhomogeneous coordinates by

$$
\zeta: \mapsto\left(\frac{\Phi_{1}(\zeta)}{\Phi_{0}(\zeta)}, \ldots, \frac{\Phi_{\frac{k-3}{2}}(\zeta)}{\Phi_{0}(\zeta)}\right) .
$$


Since $\frac{\Phi_{1}}{\Phi_{0}}$ has a simple zero at $P_{\infty}, \Phi$ is of maximal rank at this point. It is, by now, routine to reduce the study of the map $\Phi$ near an arbitrary distinguished puncture to the case where the puncture is $P_{\infty}$.

Corollary 7.3. For every prime $k \geq 7$, the map $\Phi$ is also defined by $\frac{k-1}{2}$ linearly independent abelian differentials of the first kind on $\overline{\mathbb{H}^{2} / \Gamma(k)}$.

Proof. The corollary is an immediate consequence of the previous proposition and Proposition 6.1.

Remark 10. $\Phi\left(\overline{\mathbb{H}^{2} / \Gamma(k)}\right)$ is a curve of degree $\frac{\left(k^{2}-1\right)(k-3)}{48}$ in $\mathbf{P} \mathbb{C}^{\frac{k-3}{2}}$. For $k=7$ it is a curve of genus 3 (see $\S 10$ ) and degree 4 in 2-dimensional complex projective space; as a consequence of the last corollary (or as can be seen directly $(\S 10))$, the canonical curve.

Problem 2. Is the map $\Phi$ injective?

Proposition 7.4. For each prime $k>3$, the homomorphism

$$
\Theta: \Gamma / \Gamma(k) \rightarrow \operatorname{Aut} \mathbf{P} V(k)
$$

is injective.

Proof. If $\gamma^{-1} \in \Gamma$ belongs to the kernel of $\Theta$, then there exists a nonzero complex number $\lambda$ so that for each integer $l$ with $0 \leq l \leq \frac{k-3}{2}$,

$$
\gamma_{*}\left(\varphi_{l}\right)=\lambda \varphi_{l} .
$$

By Corollary 5.4, $\gamma$ must map infinity to a $\Gamma(k)$-equivalent cusp. Thus $\gamma \in G(k)$ (see [5]), and

$$
\gamma=B^{m} \circ \gamma_{1},
$$

with $m \in \mathbb{Z}, 0 \leq m \leq k-1$, and $\gamma_{1} \in \Gamma(k)$. Since $\gamma_{*}=\left(\gamma_{1}\right)_{*} \circ\left(B_{*}\right)^{m}$ and $\gamma_{1}$ is in the kernel of $\Theta$, we conclude that $\gamma$ is in the kernel if and only if $B^{m}$ is. Using (4.3) for $l=0$ and 1 , we see that $\lambda=c(B, k)=c(B, k) \exp \left(\frac{2 \pi \imath m}{k}\right)$. Hence $m=0$.

Remark 11. It is probably true that $\Phi$ is injective. The injectivity of $\Theta$ would follow trivially from this conjecture. 


\section{8. $k=3$.}

Combining the results developed here with those of [7], we get the following identity for $\tau \in \mathbb{H}^{2}$ :

$$
\frac{\theta^{4}\left[\begin{array}{l}
\frac{1}{3} \\
1
\end{array}\right](0,3 \tau)}{\theta\left[\begin{array}{c}
1 \\
\frac{1}{3}
\end{array}\right](0, \tau) \theta\left[\begin{array}{c}
\frac{1}{3} \\
\frac{1}{3}
\end{array}\right](0, \tau) \theta\left[\begin{array}{l}
\frac{1}{3} \\
1
\end{array}\right](0, \tau) \theta\left[\begin{array}{c}
\frac{1}{3} \\
\frac{5}{3}
\end{array}\right](0, \tau)}=\frac{1}{2}+\frac{\sqrt{3}}{6} \imath .
$$

The above formula for $\theta$-constants is a special case ${ }^{15}$ of a more general relation for $\theta$-functions. For all $z \in \mathbb{C}$

$$
\begin{gathered}
\theta^{3}\left[\begin{array}{c}
\frac{1}{3} \\
1
\end{array}\right](0,3 \tau) \theta\left[\begin{array}{c}
\frac{1}{3} \\
1
\end{array}\right](3 z, 3 \tau)= \\
\left(\frac{1}{2}+\frac{\sqrt{3}}{6} \imath\right) \theta\left[\begin{array}{c}
1 \\
\frac{1}{3}
\end{array}\right](0, \tau) \theta\left[\begin{array}{c}
\frac{1}{3} \\
\frac{1}{3}
\end{array}\right](z, \tau) \theta\left[\begin{array}{c}
\frac{1}{3} \\
1
\end{array}\right](z, \tau) \theta\left[\begin{array}{c}
\frac{1}{3} \\
\frac{5}{3}
\end{array}\right](z, \tau) .
\end{gathered}
$$

We turn now to an application in number theory. Dedekind's etafunction (see, for example, [16, pg. 129])

$$
\eta(z)=z^{\frac{1}{24}} \prod_{n=1}^{n=\infty}\left(1-z^{n}\right)=\sum_{n=-\infty}^{\infty}(-1)^{n} z^{\frac{3 n^{2}+n}{2}}, z \in \mathbb{C},|z|<1
$$

arises most naturally in the theory of the partition function for the positive integers. If $N$ is a positive integer, we define $P(N)$ to be the number of ways $N$ can be expressed as a sum of nonincreasing positive integers. It is well known [10, Chapter XIX] that if we define $P(0)=1$, then

$$
\sum_{n=0}^{\infty} P(n) z^{n}=\frac{z^{\frac{1}{24}}}{\eta(z)}, z \in \mathbb{C},|z|<1 .
$$

For $\tau \in \mathbb{H}^{2}$, we set $z=\exp (2 \pi \imath \tau)$. We can hence view $\eta$ as a function on the upper half plane. It is an interesting observation, nontrivial to verify [19, pgs. 95 and 109], that $\eta$ satisfies a functional equation. It is routine to verify (by comparing Fourier series expansions) that

$$
\eta(\tau)=\exp \left(-\frac{\pi \imath}{6}\right) \theta\left[\begin{array}{c}
\frac{1}{3} \\
1
\end{array}\right](0,3 \tau), \tau \in \mathbb{H}^{2} .
$$

\footnotetext{
${ }^{15}$ The relevant theory will be developed in a forthcoming paper.
} 
We conclude from (4.3) and (4.4) that

$$
\eta(\tau+1)=\exp \left(\frac{\pi \imath}{12}\right) \eta(\tau) \text { and } \eta\left(-\frac{1}{\tau}\right)=\sqrt{-\imath \tau} \eta(\tau), \text { for all } \tau \in \mathbb{H}^{2}
$$

$$
\text { 9. } k=5 \text {. }
$$

We begin with a result that, as above, combines ideas from two different approaches. It is the identity for $\tau \in \mathbb{H}^{2}$ :

$$
\begin{aligned}
& \frac{\theta\left[\begin{array}{l}
\frac{1}{5} \\
1
\end{array}\right](0,5 \tau)}{\theta\left[\begin{array}{l}
\frac{3}{5} \\
1
\end{array}\right](0,5 \tau)}=\exp \frac{4 \pi \imath}{5} \frac{\theta\left[\begin{array}{l}
\frac{1}{5} \\
\frac{1}{5}
\end{array}\right](0, \tau) \theta\left[\begin{array}{l}
\frac{1}{5} \\
\frac{3}{5}
\end{array}\right](0, \tau)}{\theta\left[\begin{array}{c}
\frac{3}{5} \\
\frac{1}{5}
\end{array}\right](0, \tau) \theta\left[\begin{array}{l}
\frac{3}{3} \\
\frac{3}{5}
\end{array}\right](0, \tau)} \\
& \frac{\theta\left[\begin{array}{l}
\frac{1}{5} \\
1
\end{array}\right](0, \tau) \theta\left[\begin{array}{l}
\frac{1}{5} \\
\frac{7}{5}
\end{array}\right](0, \tau) \theta\left[\begin{array}{l}
\frac{1}{5} \\
\frac{9}{5}
\end{array}\right](0, \tau)}{\theta\left[\begin{array}{c}
\frac{3}{5} \\
1
\end{array}\right](0, \tau) \theta\left[\begin{array}{c}
\frac{3}{5} \\
\frac{7}{5}
\end{array}\right](0, \tau) \theta\left[\begin{array}{c}
\frac{3}{5} \\
\frac{9}{5}
\end{array}\right](0, \tau)} .
\end{aligned}
$$

We now apply the theory developed in this paper to construct a special 12 punctured sphere and a group of fractional linear transformations isomorphic to $\Gamma / \Gamma(5)$ which in turn is isomorphic to $\operatorname{PSL}\left(2, \mathbb{Z}_{5}\right)$. We shall also recover the classically known fact that this group is isomorphic to the symmetry group of the regular icosahedron [1, pg. 2].

The Riemann surface $\mathbb{H}^{2} / \Gamma(5)$ is a 12 times punctured sphere (see [7, $\S 11]$ for related results). The mapping

$$
\tau \mapsto\left(\varphi_{0}(\tau), \varphi_{1}(\tau)\right), \tau \in \mathbb{H}^{2} \cup \mathbb{Q} \cup\{\infty\},
$$

defines an embedding of $\mathbb{H}^{2} / \Gamma(5)$ into $\mathbf{P} \mathbb{C}$. This follows from the observation that for $f=f_{0}=\frac{\varphi_{0}}{\varphi_{1}}$, we have $(F)=\frac{P_{\frac{1}{5}}}{P_{\frac{2}{5}}}$.

Our formulae for the matrices $A_{*}$ and $B_{*}$ give

$$
A_{*}=\frac{1}{\sqrt{5 \imath}}\left[\begin{array}{cc}
\exp \left(\frac{\pi \imath}{10}\right)+\exp \left(-\frac{\pi \imath}{10}\right) & \exp \left(\frac{\pi \imath}{10}\right)+\exp \left(-\frac{\pi \imath}{2}\right) \\
\exp \left(\frac{\pi \imath}{2}\right)+\exp \left(-\frac{\pi \imath}{10}\right) & \exp \left(\frac{9 \pi}{10}\right)+\exp \left(-\frac{9 \pi \imath}{10}\right)
\end{array}\right]
$$

and

$$
B_{*}=c(B, k)\left[\begin{array}{cc}
1 & 0 \\
0 & \exp \left(\frac{2 \pi \imath}{5}\right)
\end{array}\right]
$$


We may identify $\Phi$ with $f$, in this case. Hence for $\gamma \in \Gamma$, we may identify $\gamma_{*}$ with $\tilde{\gamma}$.

We need to digress to discuss a metric on $\mathbf{P} \mathbb{C}^{N}, N \in \mathbb{Z}, N \geq 1$. Let $(\cdot, \cdot)$ and $\|\cdot\|$ represent the usual complex inner product and norm in $\mathbb{C}^{N+1}$. Then

$$
\|u-v\|^{2}=\|u\|^{2}+\|v\|^{2}-2 \Re(u, v) .
$$

If the equivalence classes of $u, v$ are now thought of as points in $\mathbf{P} \mathbb{C}^{N}$, we can always choose representatives which lie on the the unit ball in $\mathbb{C}^{N+1}$. We then define

$$
d(u, v)=\inf \|\tilde{u}-\tilde{v}\|
$$

where the infimum is taken over all elements $\tilde{u}, \tilde{v}$ in the unit ball of $\mathbb{C}^{N+1}$ equivalent to $u, v$ respectively. Clearly,

$$
\begin{aligned}
\inf _{\{\theta, \phi \in \mathbb{R}\}}\left\{2-2 \Re\left(e^{\imath \theta} u, e^{\imath \phi} v\right)\right\} \\
=\inf \left\{2-2 \Re\left(e^{\imath(\theta-\phi)}(u, v)\right)\right\}=2-2|(u, v)| .
\end{aligned}
$$

It is interesting to note that when $n=1$ the distance we have defined is related but not equal to the chordal metric on the sphere. If we identify the sphere with the one point compactification of the plane by stereographic projection

$$
z=x+\imath y \mapsto\left(\frac{2 x}{x^{2}+y^{2}+1}, \frac{2 y}{x^{2}+y^{2}+1}, \frac{x^{2}+y^{2}-1}{x^{2}+y^{2}+1}\right), z \in \hat{\mathbb{C}}=\mathbb{C} \cup\{\infty\},
$$

then the chordal metric is given by

$$
d\left(z_{1}, z_{2}\right)=\frac{\left|z_{1}-z_{2}\right|}{\sqrt{1+\left|z_{1}\right|^{2}} \sqrt{1+\left|z_{2}\right|^{2}}} .
$$

Under the usual identification of $\mathbf{P} \mathbb{C}$ with the Riemann sphere $\left(z_{1}, z_{2}\right) \in \mathbf{P} \mathbb{C}$ corresponds to $\frac{z_{1}}{z_{2}} \in \hat{\mathbb{C}}$. The chordal metric between $\left(z_{1}, z_{2}\right)$ and $\left(w_{1}, w_{2}\right)$ on $\mathbf{P} \mathbb{C}$ is therefore

$$
\frac{\left|\frac{z_{1}}{z_{2}}-\frac{w_{1}}{w_{2}}\right|}{\sqrt{1+\left|\frac{z_{1}}{z_{2}}\right|^{2}} \sqrt{1+\left|\frac{w_{1}}{w_{2}}\right|^{2}}} .
$$

If we assume that $\left|z_{1}\right|^{2}+\left|z_{2}\right|^{2}=\left|w_{1}\right|^{2}+\left|w_{2}\right|^{2}=1$, then the above reduces to $\left|z_{1} w_{2}-w_{1} z_{2}\right|$ which can be written as

$$
\sqrt{1-\left|z_{1} \bar{w}_{1}+z_{2} \bar{w}_{2}\right|^{2}}=\sqrt{1-\left|\left(\left(z_{1}, z_{2}\right),\left(w_{1}, w_{2}\right)\right)\right|^{2}} .
$$


Hence we see that our distance differs from the chordal distance. The common feature which is important however is that both of these distances are invariant under the unitary group.

We can now return to the special case under consideration. Write the matrix $\sqrt{5 \imath} A_{*}$ as $\left[\begin{array}{cc}z_{1} & z_{2} \\ w_{1} & w_{2}\end{array}\right]$ where, of course, $z_{2}=\overline{w_{1}}, w_{2}=-\overline{z_{1}}=-z_{1}$. Using the prescription of $[7, \S 2]$, we see that the 12 punctures on $\mathbb{H}^{2} / \Gamma(5)$ correspond to the equivalence classes in $\mathbf{P} \mathbb{C}$ of the following 12 points in $\mathbb{C}^{2}$ :

$$
\begin{gathered}
(1,0),(0,1),\left(z_{1}, w_{1}\right),\left(z_{2}, w_{2}\right), \\
\left(z_{1}, \exp \left(\frac{2 \pi \imath}{5}\right) w_{1}\right),\left(z_{2}, \exp \left(\frac{2 \pi \imath}{5}\right) w_{2}\right),\left(z_{1}, \exp \left(\frac{4 \pi \imath}{5}\right) w_{1}\right), \\
\left(z_{2}, \exp \left(\frac{4 \pi \imath}{5}\right) w_{2}\right),\left(z_{1}, \exp \left(\frac{6 \pi \imath}{5}\right) w_{1}\right),\left(z_{2}, \exp \left(\frac{6 \pi \imath}{5}\right) w_{2}\right) \\
\left(z_{1}, \exp \left(\frac{8 \pi \imath}{5}\right) w_{1}\right),\left(z_{2}, \exp \left(\frac{8 \pi \imath}{5}\right) w_{2}\right) .
\end{gathered}
$$

Under the usual identification of projective space $\mathbf{P} \mathbb{C}$ with the extended complex plane $\mathbb{C} \cup\{\infty\}$ the 12 punctures on $f\left(\mathbb{H}^{2} \cup \mathbb{Q} \cup\{\infty\}\right)$ are:

$$
\begin{gathered}
\infty, 0, \frac{z_{1}}{w_{1}}, \frac{z_{2}}{w_{2}}, \\
\exp \left(\frac{-2 \pi \imath}{5}\right) \frac{z_{1}}{w_{1}}, \exp \left(\frac{-2 \pi \imath}{5}\right) \frac{z_{2}}{w_{2}}, \exp \left(\frac{-4 \pi \imath}{5}\right) \frac{z_{1}}{w_{1}}, \exp \left(\frac{-4 \pi \imath}{5}\right) \frac{z_{2}}{w_{2}}, \\
\exp \left(\frac{-6 \pi \imath}{5}\right) \frac{z_{1}}{w_{1}}, \exp \left(\frac{-6 \pi \imath}{5}\right) \frac{z_{2}}{w_{2}}, \exp \left(\frac{-8 \pi \imath}{5}\right) \frac{z_{1}}{w_{1}}, \exp \left(\frac{-8 \pi \imath}{5}\right) \frac{z_{2}}{w_{2}} .
\end{gathered}
$$

Pairs of antipodal points on the sphere are given as $z$ and $\frac{-1}{\bar{z}}$. Thus the 12 punctures come in pairs of antipodal points. Furthermore, the five points of absolute value $\left|\frac{z_{i}}{w_{i}}\right|, i=1,2$ form a pentagon $\mathcal{P}_{\rangle}$. The pentagon $\mathcal{P}_{\in}\left(\mathcal{P}_{\infty}\right)$ is inside (outside) the unit circle. It is obvious that $\tilde{B}$ fixes 0 and $\infty$ and permutes the vertices of each of the pentagons. We view $\hat{\mathbb{C}}$ as the unit sphere in $\mathbb{R}^{3}$. If the vertices of each of the pentagons are joined by a straight line in $\mathbb{R}^{3}$ and then the vertices of $\mathcal{P}_{\in}\left(\mathcal{P}_{\infty}\right)$ are connected by a straight line with the origin $(\infty)$, we obtain 10 triangles; 10 of the 20 faces of the icosahedron. The remaining 10 faces are obtained by suitably joining the the vertices of the pentagon around $\infty$ with those of the pentagon around the origin. In fact the entire construction can be summarized by joining the points of minimal distance either in the chordal metric or in the metric 
we have defined above. The triangles we obtain in this way are equilateral triangles because the transformations $\tilde{\gamma}, \gamma \in \Gamma$, are isometries in the metric under consideration.

$$
\text { 10. } k=7 \text {. }
$$

\subsection{The function field $\mathcal{K}\left(\overline{\mathbb{H}^{2} / \Gamma(7)}\right)$.}

Using the 3 modified theta constants $\varphi_{0}, \varphi_{1}$ and $\varphi_{2}$, we construct 3 $\Gamma(7)$-automorphic functions

$$
f_{0}=\frac{\varphi_{0}}{\varphi_{2}}, f_{1}=\frac{\varphi_{1}}{\varphi_{2}}, f_{2}=\frac{\varphi_{0}}{\varphi_{1}} .
$$

The divisors of these functions are easily computed.

Proposition 10.1. We have

$$
\left(F_{0}\right)=\frac{P_{\frac{2}{7}}^{2} P_{\frac{3}{7}}}{P_{\infty}^{3}},\left(F_{1}\right)=\frac{P_{\frac{3}{7}}^{3}}{P_{\infty}^{2} P_{\frac{2}{7}}} \text { and }\left(F_{2}\right)=\frac{P_{\frac{2}{7}}^{3}}{P_{\infty} P_{\frac{3}{7}}^{2}} .
$$

We use $z=F_{0}$ and $w=F_{1}$ and study the correseponding holomorphic map

$$
\varphi: \overline{\mathbb{H}^{2} / \Gamma(7)} \rightarrow \mathfrak{M}^{*} .
$$

We know that in this case (see Proposition 2.1),

$$
3=\operatorname{deg} z=(\operatorname{deg} \varphi)(\operatorname{deg} \text { proj}) .
$$

Now $\varphi\left(P_{\frac{2}{7}}\right)$ is a Puiseaux series of the form

$$
\sum_{i=-1}^{\infty} c_{i} z^{\frac{i}{2}}, c_{-1} \neq 0
$$

while $\varphi\left(P_{\frac{3}{7}}\right)$ is of the form

$$
\sum_{i=3}^{\infty} c_{i} z^{i}, c_{3} \neq 0
$$

which implies that proj is nonconstant. Hence $\operatorname{deg} \operatorname{proj}=3$ and $\operatorname{deg} \varphi=1$. We hence conclude that the meromorphic functions $F_{0}$ and $F_{1}$ generate 
$\mathcal{K}\left(\overline{\mathbb{H}^{2} / \Gamma(7)}\right)$. (We could also conclude this from Theorem 6.3.) It also follows immediately that $P_{\infty}$ is a Weierstrass point on the compactified surface $\overline{\mathbb{H}^{2} / \Gamma(7)}$. Since the automorphism group acts transitively on the punctures in $\mathbb{H}^{2} / \Gamma(7)$, we conclude that each puncture is a simple Weierstrass point.

We can now take appropriate products; the projections to $\overline{\mathbb{H}^{2} / \Gamma(7)}$ of functions $f_{0}^{2} f_{2}, f_{0} f_{1}^{2}$ have divisors $\frac{P_{2}^{7}}{P_{\infty}^{7}}, \frac{P_{\frac{3}{7}}^{7}}{P_{\infty}^{7}}$.

From the definitions of the functions we know that

$$
f_{0}^{2} f_{2}=\frac{\varphi_{0}^{3}}{\varphi_{1} \varphi_{2}^{2}}, f_{0} f_{1}^{2}=\frac{\varphi_{0} \varphi_{1}^{2}}{\varphi_{2}^{3}}
$$

Both of the functions in question are lifts of univalent functions on $\mathbb{H}^{2} / G(7)$ with the pole at the same point (their divisors are $\frac{P_{2}}{P_{\infty}}$ and $\frac{P_{3}}{P_{\infty}}$, respectively); which implies that one of these two functions is obtained from the other by post composition by an affine map. This affine map is easily computed to yield the identity

$$
\frac{\varphi_{0}^{3}}{\varphi_{1} \varphi_{2}^{2}}=\epsilon^{-1} \frac{\varphi_{0} \varphi_{1}^{2}}{\varphi_{2}^{3}}+\epsilon^{2}, \epsilon=\exp \left(\frac{\pi \imath}{7}\right)
$$

which we can rewrite as

$$
\begin{gathered}
\left(\omega^{-3} \theta\left[\begin{array}{c}
\frac{1}{7} \\
1
\end{array}\right]\right)^{3}\left(\omega^{9} \theta\left[\begin{array}{c}
\frac{5}{7} \\
1
\end{array}\right]\right)+\left(\omega^{-3} \theta\left[\begin{array}{l}
\frac{1}{7} \\
1
\end{array}\right]\right)\left(\omega^{9} \theta\left[\begin{array}{l}
\frac{3}{7} \\
1
\end{array}\right]\right)^{3} \\
+\left(\omega^{9} \theta\left[\begin{array}{c}
\frac{3}{7} \\
1
\end{array}\right]\right)\left(\omega^{9} \theta\left[\begin{array}{c}
\frac{5}{7} \\
1
\end{array}\right]\right)^{3}=0, \omega=\exp \left(\frac{\pi \imath}{28}\right) .
\end{gathered}
$$

The above identity is also deriveable abstractly from the theory of [4] (see also Theorem 15.4), and it tells us that the Riemann surface $\overline{\mathbb{H}^{2} / \Gamma(7)}$ is realizeable as the locus $X^{3} Z+Y^{3} X+Z^{3} Y=0$ in $\mathbf{P} \mathbb{C}^{2}$. Alternatively, starting with the functions $F_{0}^{2} F_{2}$ and $F_{0}$, we can describe $\overline{\mathbb{H}^{2} / \Gamma(7)}$ as the Riemann surface of the algebraic equation $w^{7}=z^{2}(z-1)$. Furthermore, we have realized its automorphism group as a subgroup of the projective linear maps of $\mathbf{P} \mathbb{C}^{2}$.

\subsection{The projective embedding of $\overline{\mathbb{H}^{2} / \Gamma(7)}$.}

We use the two degree 3 functions $F_{0}$ and $F_{1}$ to embedd $\overline{\mathbb{H}^{2} / \Gamma(7)}$ into $\mathbf{P} \mathbb{C}^{2}$ by the map

$$
\varphi: Q \mapsto\left(F_{0}(Q), F_{1}(Q), 1\right)
$$


We claim that this map $\varphi$ is injective. We first observe that for the 3 distinguished punctures, we have in homogeneous coordinates

$$
\varphi\left(P_{\infty}\right)=(1,0,0), \varphi\left(P_{\frac{2}{7}}\right)=(0,1,0) \text { and } \varphi\left(P_{\frac{3}{7}}\right)=(0,0,1),
$$

while for $Q \in \mathbb{H}^{2} / \Gamma(7)$ or $Q$ a nondistinguished puncture on this surface, $\varphi(Q)$ never lands in a coordinate hyperplane (where one of the three homogeneous coordinates vanishes). Thus if

$$
\left(F_{0}\left(Q_{1}\right), F_{1}\left(Q_{1}\right), 1\right)=\left(F_{0}\left(Q_{2}\right), F_{1}\left(Q_{2}\right), 1\right)
$$

for distinct $Q_{1}$ and $Q_{2}$ in $\overline{\mathbb{H}^{2} / \Gamma(7)}$, then for $F_{i}\left(Q_{1}\right)=z_{i} \in \mathbb{C}^{*}, i=0,1$, we have (as divisors) $F_{0}^{-1}\left(z_{1}\right)=Q_{1} Q_{2} R_{1}$ and $F_{1}^{-1}\left(z_{2}\right)=Q_{1} Q_{2} R_{2}$ for some points $R_{1}$ and $R_{2}$ in $\mathbb{H}^{2} / \Gamma(7)$. The surface $\overline{\mathbb{H}^{2} / \Gamma(7)}$ has genus 3 and is not hyperelliptic (see, for example, [6, Proposition III.7.10]). Hence

$$
i\left(Q_{1} Q_{2}\right)=i\left(P_{\infty}^{2}\right)=i\left(P_{\infty}^{3}\right)=i\left(P_{\infty}^{2} P_{\frac{2}{7}}\right)=1 .
$$

We have seen that

$$
r\left(\frac{1}{P_{\infty}^{3} P_{\frac{2}{7}}}\right) \geq 3 .
$$

Riemann-Roch then tells us that

$$
i\left(P_{\infty}^{3} P_{\frac{2}{7}}\right)=1 .
$$

The divisor $F_{0}^{-1}\left(z_{1}\right)=Q_{1} Q_{2} R_{1}$ is equivalent $(\sim)$ to $F_{0}^{-1}(\infty)=P_{\infty}^{3}$. This implies that $Q_{1} Q_{2} R_{1} P_{\frac{2}{7}} \sim P_{\infty}^{3} P_{\frac{2}{7}}$ which is canonical. In the same way we see that $Q_{1} Q_{2} R_{2} P_{\infty} \sim P_{\frac{3}{7}}^{3} P_{\infty}$ is canonical. This tells us that $R_{1} P_{\frac{2}{7}}=R_{2} P_{\infty}$, an obvious contradiction.

We already know that $\Phi$ is of maximal rank at all the distinguished punctures. The above argument with $Q_{1}=Q_{2}$ shows that $\Phi$ is globally of maximal rank. Hence $\Phi\left(\overline{\mathbb{H}^{2} / \Gamma(7)}\right)$ is nonsingular. Further, every hyperplane in $\mathbf{P} \mathbb{C}^{2}$ intersects $\Phi\left(\overline{\mathbb{H}^{2} / \Gamma(7)}\right)$ in 4 points counting multiplicities. By [6, Theorem III.10.5], $\Phi\left(\overline{\mathbb{H}^{2} / \Gamma(7)}\right) \subset \mathbf{P} \mathbb{C}^{2}$ is canonical. Computing the intersection of image curve with the hyperplane where the first coordinate equals to zero, we conclude that

$$
i\left(P_{\frac{2}{7}}^{3} P_{\frac{3}{7}}\right)=1 .
$$

Similarly $P_{\frac{3}{7}}^{3} P_{\frac{1}{7}}$ and (as we already know) $P_{\frac{1}{7}}^{3} P_{\frac{2}{7}}$ are canonical divisors. We have established a seemingly odd duality between $V(7)$ and the vector space 
$\mathcal{H}$ of abelian differentials of the first kind on the compact Riemann surface $\overline{\mathbb{H}^{2} / \Gamma(7)}$ leading to a construction of all the integral canonical divisors on this surface. The construction goes as follows. Choose a nonzero $\varphi \in V(7)$. Divide the divisor $(\varphi)$ by the $\frac{1}{8}$ power of the clivisor $D$ of $\Gamma(7)$-inequivalent cusps in $\mathbb{Q} \cup\{\infty\}$. The projection of this divisor to the surface $\overline{\mathbb{H}^{2} / \Gamma(7)}$ is an integral canonical divisor on this surface, and conversely every canonical integral divisor on $\overline{\mathbb{H}^{2} / \Gamma(7)}$ is so obtained. It is natural to seek an explanation of this phenomenon. We offer two possible explanations.

Choose a generic element $f_{o} \in V(7)$. The function $f_{o}$ will have 4 inequivalent simple zeros at points in $\mathbb{H}^{2}$ and a zero of order $\frac{1}{8}$ at each cusp. A ratio $\frac{\varphi}{f_{o}}$ with $\varphi \in V(7)$ will project to $F$, a constant function or a function of degree 3 or 4 on $\overline{\mathbb{H}^{2} / \Gamma(7)}$. In the case of a degree 4 function, $(F)$ will be canonical (see Proposition 6.1 and Remark 9). Choosing a holomorphic 1differential $\omega$ on $\overline{\mathbb{H}^{2} / \Gamma(7)}$, we see that an arbitrary holomorphic 1-differential is given as $\omega F$. For the second explanation, let $W$ be the lift to $\mathbb{H}^{2}$ of a Wronskian of a basis for $\mathcal{H}$. The divisor of the 6 -form $W$ consists of the Weierstrass points of $\overline{\mathbb{H}^{2} / \Gamma(7)}$ and hence equals $D$. The map $\Phi$ of $\overline{\mathbb{H}^{2} / \Gamma(7)}$ into $\mathbf{P} \mathbb{C}^{2}$ is defined by the two triples $\left(\varphi_{0}, \varphi_{1}, \varphi_{2}\right)$ and $W^{\frac{1}{8}}\left(\varphi_{0}, \varphi_{1}, \varphi_{2}\right)$. One easily sees that $W^{\frac{1}{8}} \varphi_{l}, l=0,1,2$, projects to a holomorphic 1 -form on $\overline{\mathbb{H}^{2} / \Gamma(7)}$. Alternately, we have seen that $\Phi$ is defined by the 3 functions $\left(F_{0}, F_{1}, 1\right)$. We know that the divisor $P_{\infty}^{3} P_{\frac{2}{7}}$ is canonical. We can hence choose a holomorphic 1-form $\omega$ on $\overline{\mathbb{H}^{2} / \Gamma(7)}$ with $(\omega)=P_{\infty}^{3} P_{\frac{2}{7}}$ and conlude that $\Phi$ is defined by the 3 linearly independent holomorphic 1-forms $\omega\left(F_{0}, F_{1}, 1\right)$, as expected from Corollary 7.3.

Remark 12. The injectivity of the map $\varphi$ implies at once that $\mathcal{K}\left(\overline{\mathbb{H}^{2} / \Gamma(7)}\right)$ is generated by $F_{1}$ and $F_{2}$. That a primitive pair does not lead to a projective embedding is illustrated in the next section.

$$
\text { 11. } k=11 \text {. }
$$

In this case, $k=11$, we are dealing with a compact surface of genus 26 punctured at 60 points. The book-keeping can already be a bit troublesome. Using the 5 modified theta constants $\varphi_{i}, i=0,1, \ldots, 4$, we construct 4 
$\Gamma(11)$-automorphic functions $f_{i}=\frac{\varphi_{i}}{\varphi_{4}}, i=0,1,2,3$. Their divisors are

$$
\begin{aligned}
& \left(F_{0}\right)=\frac{P_{\frac{2}{11}}^{4} P_{\frac{4}{11}}^{5} P_{\frac{5}{11}}^{3}}{P_{\infty}^{10} P_{\frac{3}{11}}^{2}},\left(F_{1}\right)=\frac{P_{\frac{3}{11}}^{7} P_{\frac{5}{11}}^{6}}{P_{\infty}^{9} P_{\frac{2}{11}}^{3} P_{\frac{4}{11}}}, \\
& \left(F_{2}\right)=\frac{P_{\frac{3}{11}}^{3} P_{\frac{4}{11}}^{9} P_{\frac{5}{11}}}{P_{\infty}^{7} P_{\frac{2}{11}}^{6}},\left(F_{3}\right)=\frac{P_{\frac{4}{11}}^{2} P_{\frac{5}{11}}^{10}}{P_{\infty}^{4} P_{\frac{2}{11}}^{5} P_{\frac{3}{11}}^{3}} .
\end{aligned}
$$

The material in $\S 2.3$ now shows that $z=F_{0}$ and $w=F_{3}$ generate $\mathcal{K}\left(\overline{\mathbb{H}^{2} / \Gamma(11)}\right)$, and the formulae in $\S 6.2$ show that the two functions on $\mathbb{H}^{2}$

$$
\frac{\varphi_{0}^{2}}{\varphi_{1} \varphi_{4}}=\frac{f_{0}^{2}}{f_{1}} \text { and } \frac{\varphi_{0} \varphi_{3}}{\varphi_{2}^{2}}=\frac{f_{0} f_{3}}{f_{2}^{2}}
$$

project to functions of degree 2 on the torus $\mathbb{H}^{2} / G(11)$ whose divisors are

$$
\frac{P_{\frac{2}{11}} P_{\frac{4}{11}}}{P_{\infty} P_{\frac{3}{11}}} \text { and } \frac{P_{\frac{2}{11}} P_{\frac{5}{11}}}{P_{\frac{3}{11}} P_{\frac{4}{11}}},
$$

respectively. Even though the meromorphic functions $F_{0}$ and $F_{3}$ generate the function field on the surface $\overline{\mathbb{H}^{2} / \Gamma(11)}$, they do not embedd this surface into $\mathbf{P} \mathbb{C}^{2}$ because the two punctures $P_{\frac{2}{11}}$ and $P_{\frac{3}{11}}$ are sent by the map $\left(1, F_{0}, F_{3}\right)$ to $(0,0,1)$. Note that $F_{0} F_{1} \in L\left(P_{\infty}^{-19}\right)-L\left(P_{\infty}^{-18}\right)$; so that all the punctures are Weierstrass points and the lowest "non-gap" is at most 19. The fact that the punctures are all Weierstrass points comes as no surprise since there is an automorphism of the surface which has more than four fixed points (all the distinguished punctures). We do not understand why the Weierstrass "non-gap" sequence contains 19 , nor do we know if we can produce a function with a single pole of lower degree. The map $\Phi$ is defined by linearly independend holomorphic 1 -forms ${ }^{16}$. To see this, choose a holomorphic 1-form $\omega$ whose divisor is a multiple of $P_{\infty}^{10} P_{\frac{2}{11}}^{6} P_{\frac{3}{11}}^{3} P_{\frac{4}{11}}$, and observe that $\omega\left(F_{0}, F_{1}, F_{2}, F_{3}, 1\right)$ also defines $\Phi$. We have once again used Corollary 7.3.

$$
\text { 12. } k=13 \text {. }
$$

It was shown in [5] that $\overline{\mathbb{H}^{2} / G(13)}$ is a closed surface of genus 2; hence hyperelliptic (see also [20, Proposition 1.43]). The function

$$
z=\frac{\varphi_{5} \varphi_{1}}{\varphi_{2} \varphi_{0}}
$$

\footnotetext{
${ }^{16}$ In contrast to the $k=7$ case, not a maximal set of such forms.
} 
is $G(13)$-invariant; a straightforward calculation shows that $z$ is also invariant under the elliptic transformation (of order 2)

$$
\gamma=\left[\begin{array}{cc}
5 & -2 \\
13 & -5
\end{array}\right] \in \Gamma_{o}(13)
$$

On $\overline{\mathbb{H}^{2} / G(13)}$,

$$
(Z)=\frac{P_{\frac{1}{13}} P_{\frac{5}{13}}}{P_{\frac{4}{13}} P_{\frac{6}{13}}} .
$$

We conclude that on $\mathbb{S}=\overline{\mathbb{H}^{2} /\langle G(13), \gamma\rangle}$ we have a function of degree one (with a simple zero at $P_{\frac{1}{13}}$ and a simple pole at $P_{\frac{4}{13}}$ ). Hence $\mathbb{S}$ has genus zero, and $\gamma$ induces the hyperelliptic involution on $\overline{\mathbb{H}^{2} / G(13)}(Z$ is a function of degree 2 on this surface and a function of degree one on $\mathbb{S})$. Moreover, the 6 fixed points of $\tilde{\gamma}$ are the Weierstrass points on $\overline{\mathbb{H}^{2} / G(13)}$. We can easily find one fixed point (in $\left.\mathbb{H}^{2}\right) z_{o}=\frac{5+2}{13}$ of $\gamma$; its projection to $\overline{\mathbb{H}^{2} / G(13)}$ is fixed by the hyperelliptic involution. To find the other fixed points, we introduce the motion

$$
\delta=\left[\begin{array}{cc}
2 & 1 \\
13 & 7
\end{array}\right] \in \Gamma_{o}(13)
$$

that defines an automorphism of order 6 of $\overline{\mathbb{H}^{2} / G(13)}$. Since $\delta^{3} \circ \gamma^{-1} \in G(13)$, $\delta^{3}$ also induces the hyperelliptic involution on $\overline{\mathbb{H}^{2} / G(13)}$. The projections of the 3 points $\delta^{j-1}\left(z_{o}\right), j=1,2,3$, are 3 of the 6 Weierstrass points on $\overline{\mathbb{H}^{2} / G(13)}$. It is not clear how to locate the other 3 .

We have produced a meromorphic function $(Z)$ of degree 26 on the closed surface $\overline{\mathbb{H}^{2} / \Gamma(13)}$ of genus 50. It is not clear whether $\overline{\mathbb{H}^{2} / \Gamma(13)}$ carries a function of lower degree, nor is it obvious how to generalize the material of this section to primes $>13$. The material of this section hints at the complexities involved in studying the general case.

$$
\text { 13. } k=9 \text {. }
$$

The purpose of this section is to illustrate what goes wrong when $k$ is not prime and to hint at the possiblities in the study of the composite case. The case $k=9$ corresponds to a compact surface $X$ of genus 10 punctured at 36 points. The punctures correspond to the characteristics in $X_{o}(9)$ and are given concretely as the images under $P: \mathbb{H}^{2} \cup \mathbb{Q} \cup\{\infty\} \rightarrow$ $X=\left(\mathbb{H}^{2} \cup \mathbb{Q} \cup\{\infty\}\right) / \Gamma(9)$ of the rational numbers

$$
0,1,2,3,4,5,6,7,8, \frac{1}{2}, \frac{3}{2}, \frac{5}{2}, \ldots, \frac{17}{2},
$$




$$
\frac{1}{3}, \frac{2}{3}, \frac{4}{3}, \frac{5}{3}, \frac{7}{3}, \frac{8}{3}, \frac{1}{4}, \frac{3}{4}, \frac{5}{4}, \ldots, \frac{17}{4}, \frac{1}{9}, \frac{2}{9}, \frac{4}{9} .
$$

The techniques we have developed for the prime case (§5.1) will allow us to determine the orders of divisors of forms and functions at 30 of the punctures of $\mathbb{H}^{2} / \Gamma(9)$; the general theory ( $(55.2)$ is needed for the remaining 6 punctures (the projections of the first 6 rationals in the second row above). The punctures on $\mathbb{H}^{2} / \Gamma(9)$ fall into 4 disjoint sets [20, Proposition 1.43]: (1) those $\Gamma_{o}(9) / \Gamma(9)$-equivalent to $P_{\infty}\left(P_{\frac{1}{9}}, P_{\frac{2}{9}}, P_{\frac{4}{9}}\right),(2)$ those $\Gamma_{o}(9) / \Gamma(9)$-equivalent to $P_{\frac{1}{3}}\left(P_{\frac{1}{3}}, P_{\frac{4}{3}}, P_{\frac{7}{3}}\right),(3)$ those $\Gamma_{o}(9) / \Gamma(9)$-equivalent to $P_{\frac{2}{3}}\left(P_{\frac{2}{3}}, P_{\frac{5}{3}}, P_{\frac{8}{3}}\right)$, and (4) those $\Gamma_{o}(9) / \Gamma(9)$-equivalent to $P_{0}$ (the remaining 27 punctures). Because of (5.1) and the fact that the divisor of each $\varphi_{l}$ has degree $\frac{27}{2}$, we need only compute, in addition to the answers provided by Lemma $5.1, \operatorname{ord}_{x} \varphi_{l}$ for $x=\frac{1}{3}, \frac{2}{3}, l=0, \ldots, 3$. The results are summarized in the following table with entries $8 \operatorname{ord}_{x} \varphi_{l}$. The calculations at $x=\frac{1}{3}$ and $\frac{2}{3}$ can be based on the fact that $C_{1}=A \circ B^{-3} \circ A=\left[\begin{array}{ll}1 & 0 \\ 3 & 1\end{array}\right]$ and $C_{2}=B \circ A \circ B^{3} \circ A=\left[\begin{array}{ll}2 & -1 \\ 3 & -1\end{array}\right]$ map $\infty$ to these points, respectively. It follows that the matrices $\left(C_{1}\right)_{*}$ and $\left(C_{2}\right)_{*}$ are both of the form (the blank spots represent nonzero entries)

$$
\left[\begin{array}{llll} 
& 0 & & \\
0 & & 0 & 0 \\
& 0 & & \\
& 0 & &
\end{array}\right] .
$$

Values of $8 \operatorname{ord}_{x} \varphi_{l}$

$$
\begin{array}{rrrrr}
\operatorname{cusp} x & l=0 & \underline{l}=1 & \underline{l}=2 & \underline{l=3} \\
\frac{1}{9} & 1 & 9 & 25 & 49 \\
\frac{2}{9} & 49 & 9 & 1 & 25 \\
\frac{4}{9} & 25 & 9 & 49 & 1 \\
0, \ldots, 8, \frac{1}{2}, \ldots, \frac{17}{2}, \frac{1}{4}, \ldots, \frac{1}{4}, \frac{17}{4} & 1 & 1 & 1 & 1
\end{array}
$$

We are dealing with the map

$$
\Phi: \tau \mapsto\left(\varphi_{0}(\tau), \varphi_{1}(\tau), \varphi_{2}(\tau), \varphi_{3}(\tau)\right), \tau \in \mathbb{H}^{2} \cup \mathbb{Q} \cup\{\infty\} .
$$

As with primes, we can compute the images under $\Phi$ of the cusps $\Gamma_{o}(k)$ equivalent to $\infty$. We see that

$$
P_{\frac{1}{9}} \mapsto(1,0,0,0), P_{\frac{2}{9}} \mapsto(0,0,1,0), \text { and } P_{\frac{4}{9}} \mapsto(0,0,0,1)
$$


The last table also shows that $(0,1,0,0) \notin \Phi\left(\overline{\mathbb{H}^{2} / \Gamma(9)}\right)$. The map $\Phi$ is also defined by the meromorphic functions $\left(F_{0}, F_{1}, F_{2}, 1\right)$ and the holomorphic 1-forms $\omega\left(F_{0}, F_{1}, F_{2}, 1\right)$, where $\omega$ is a holomorphic differential on $\overline{\mathbb{H}^{2} / \Gamma(9)}$ whose divisor is a multiple of $P_{\infty}^{6} P_{\frac{2}{9}}^{3}$. Since the surface $\overline{\mathbb{H}^{2} / \Gamma(9)}$ has genus 10 , there is always such a $\omega$. More information on the map $\Phi$ is given in the proof of Theorem 15.5.

We have observed that for primes $k$, the support of the divisor of any quotient $F=\frac{\Phi_{l}}{\Phi_{l^{\prime}}}$ is precisely the set of distinguished punctures,

$$
\left\{P_{\frac{1}{k}}, P_{\frac{2}{k}}, \ldots, P_{\frac{k-1}{2}}\right\}
$$

This fails ${ }^{17}$ for $k=9$ as illustrated by $l=1, l^{\prime}=3$. We find that in this case the function $F$ has a pole of order 5 at $P_{\frac{1}{9}}=P_{\infty}$ and a pole of order 2 at $P_{\frac{2}{9}}$. If the support of the divisor $(F)$ were at the 3 distinguished punctures, then $F$ would be forced to have a zero of order 7 at the point $P_{\frac{4}{9}}$. We find however that $F$ indeed has a zero at $P_{\frac{4}{9}}$ but only a simple zero. It follows that the function $F$ has at least 6 additional zeros. The above table allows us to conclude that $F$ has simple zeros at the 6 points $\left\{P_{\frac{1}{3}}, P_{\frac{2}{3}}, P_{\frac{4}{3}}, P_{\frac{5}{3}}, P_{\frac{7}{3}}, P_{\frac{8}{3}}\right\}$, and is regular elsewhere. We summarize the above and related calculations (for $l=0, l^{\prime}=3$ and for $l=2, l^{\prime}=3$ ) in

$$
\left(\frac{\Phi_{1}}{\Phi_{3}}\right)=\frac{P_{\frac{4}{9}} P_{\frac{1}{3}} P_{\frac{2}{3}} P_{\frac{4}{3}} P_{\frac{5}{3}} P_{\frac{7}{3}} P_{\frac{8}{3}}}{P_{\frac{1}{9}}^{5} P_{\frac{2}{9}}^{2}},\left(\frac{\Phi_{0}}{\Phi_{3}}\right)=\frac{P_{\frac{2}{9}}^{3} P_{\frac{4}{9}}^{3}}{P_{\frac{1}{9}}^{6}} \text { and }\left(\frac{\Phi_{2}}{\Phi_{3}}\right)=\frac{P_{\frac{4}{9}}^{6}}{P_{\frac{1}{9}}^{3} P_{\frac{2}{9}}^{3}} \text {. }
$$

Since we have produced a meromorphic function of degree 6 on $X$ which is holomorphic on $X-\left\{P_{\infty}\right\}$, we conclude that $P_{\infty}$ is a Weierstrass point and thus all 36 punctures on $\mathbb{H}^{2} / \Gamma(9)$ are Weierstrass points. In fact we can determine 8 of the first 10 "non-gaps" at $P_{\infty}$; they are: $6,9,11,12,15,17$, $18,20$.

There is an additional observation to be made. We consider in place of $\Phi$, the map

$$
\Phi_{1}: \tau \mapsto\left(\varphi_{0}(\tau), \varphi_{2}(\tau), \varphi_{3}(\tau)\right), \tau \in \mathbb{H}^{2} \cup \mathbb{Q} \cup\{\infty\} .
$$

The reader can check that the mapping in this case is certainly not injective, and in fact that here we have a map from $Y=\overline{\mathbb{H}^{2} / \Gamma(9) / G}$ to $\mathbf{P} \mathbb{C}^{2}$, where

${ }^{17}$ Recall that for $k=9$, we have only 3 distinguished punctures: $\left\{P_{\frac{1}{9}}, P_{\frac{2}{9}}, P_{\frac{4}{9}}\right\}$. 
$G$ is the group generated by $\tilde{B}^{3}$. Now $\tilde{B}^{3}$ is an automorphism of $\mathbb{H}^{2} / \Gamma(9)$ of period 3 with exactly 9 fixed points, all at the punctures of $\mathbb{H}^{2} / \Gamma(9)$. The fixed points are the images under $P$ of the points

$$
\frac{1}{3}, \frac{2}{3}, \frac{4}{3}, \frac{5}{3}, \frac{7}{3}, \frac{8}{3}, \frac{1}{9}, \frac{2}{9}, \frac{4}{9}
$$

and the quotient $\mathbb{H}^{2} / \Gamma(9) / G$ is a torus with 18 punctures.

The equations of $X$ and $Y$ will be given at the end of this paper as an example of our general theory. We now use the functions $z=\frac{\Phi_{0}}{\Phi_{3}}$ and $w=\frac{\Phi_{3}}{\Phi_{2}}$ on $Y$. The divisors of these functions are

$$
(z)=\frac{P_{\frac{2}{9}} P_{\frac{4}{9}}}{P_{\frac{1}{9}}^{2}} \text { and }(w)=\frac{P_{\frac{1}{9}} P_{\frac{2}{9}}}{P_{\frac{4}{9}}^{2}} .
$$

For the resulting map $\varphi$, we have $(\operatorname{proj} \circ \varphi)\left(P_{\frac{2}{9}}\right)=0=(\operatorname{proj} \circ \varphi)\left(P_{\frac{4}{9}}\right)$. Hence by Proposition 2.1, $\varphi$ is injective. Since $B$ induces an automorphism of period 3 of $Y$ with a fixed point, $Y$ is conformally equivalent to $T_{\frac{1}{2}+2 \frac{\sqrt{3}}{2}}$. It might be interesting to determine the involution with the same fixed point as $\tilde{B}$.

We return to a study of the two maps into projective space. The map $\Phi_{1}: Y \rightarrow \mathbf{P} \mathbb{C}^{2}$ is injective ${ }^{18}$ and of maximal rank. For if not, we can derive a linear relation

$$
c_{1} \varphi_{0} \varphi_{2}+c_{2} \varphi_{0} \varphi_{3}+c_{3} \varphi_{2} \varphi_{3}+c_{4} \varphi_{3}^{2}=0, c_{i} \in \mathbb{C}, i=1,2,3,4
$$

which is seen to be a contradiction by examining the orders of the zeros at $\infty$ of the functions appearing in the above equation. Since the map $\Phi_{1}$ may be viewed as the map $\Phi$ followed by a projection ${ }^{19}$, we conclude at once that $\Phi$ is of maximal rank. As a map from $\overline{\mathbb{H}^{2}} / \Gamma(9)$, it is injective. For if $\Phi(x)=\Phi(y)$ with $x$ and $y$ in $\overline{\mathbb{H}^{2} / \Gamma(9)}$, then $\Phi_{1}\left(x_{1}\right)=\Phi_{1}\left(y_{1}\right)$, where $x_{1}$ and $x_{2}$ are the projections of $x$ and $y$ under the canonical map $\overline{\mathbb{H}^{2} / \Gamma(9)}=X \rightarrow Y$. It follows that $y=\tilde{B}^{3 j}$ with $j=0,1$, or 2 . As a consequence of (4.3), $j=0$.

\footnotetext{
${ }^{18}$ This assertion implies at once that the map $\varphi$ is injective.

${ }^{19}$ This assertion follows from the fact for each cusp $x \in \mathbb{Q} \cup\{\infty\}$, there is at least one $j=0,2$, or 4 , such that

$$
\operatorname{ord}_{x} \varphi_{1} \geq \operatorname{ord}_{x} \varphi_{j}
$$
}




\section{The function field of $\overline{\mathbb{H}^{2} / \Gamma(k)}$ over $\overline{\mathbb{H}^{2} / \Gamma}$.}

The following theorem is an immediate corollary of a result of E. Artin. It is independent of Theorem 6.3.

Theorem 14.1. For each prime $k \geq 5$, the meromorphic functions

$$
f_{l}=\frac{\varphi_{l}}{\varphi_{\frac{k-3}{2}}}, l=0,1, \ldots, \frac{k-5}{2}
$$

generate the function field of $\overline{\mathbb{H}^{2} / \Gamma(k)}$ over $\overline{\mathbb{H}^{2}} / \Gamma$. Furthermore, $\mathcal{K}\left(\overline{\mathbb{H}^{2} / \Gamma(k)}\right.$ is a Galois extension of $\mathcal{K}\left(\overline{\left.\mathbb{H}^{2} / \Gamma\right)}\right.$ with Galois group $\Gamma / \Gamma(k)$.

Proof. Recall that the $\jmath$-invariant generates the function field $\mathcal{K}\left(\overline{\mathbb{H}^{2}} / \bar{\Gamma}\right)$ of $\overline{\mathbb{H}^{2} / \Gamma}$; that is, every meromorphic function on the Riemann sphere $\overline{\mathbb{H}^{2} / \Gamma}$ is a rational function of $\jmath$. Let $\mathbb{K}$ denote the field over $\mathbb{C}$ generated by $\jmath$ and the $\frac{k-3}{2}$ functions listed above. The group $\Gamma / \Gamma(k) \cong \operatorname{PSL}\left(2, \mathbb{Z}_{k}\right)$ acts as a group of automorphisms of $\mathbb{K}$ as a consequence of Proposition 7.4; its fixed field is precisely $\mathbb{C}(\jmath)$. By a theorem E. Artin in [15, page 194], $\mathbb{K}$ is a Galois extension of $\mathcal{K}\left(\overline{\mathbb{H}^{2}} / \Gamma\right)$ of degree $|\Gamma / \Gamma(k)|$. So is, $\mathcal{K}\left(\overline{\mathbb{H}^{2}} / \Gamma(k)\right)$. Since $\mathbb{K} \subset \mathcal{K}\left(\overline{\mathbb{H}^{2} / \Gamma(k)}\right)$, we conclude that $\mathbb{K}=\mathcal{K}\left(\overline{\mathbb{H}^{2} / \Gamma(k)}\right)$.

Remark 13. As a consequence of Theorem 6.4, the single function $w=\frac{\varphi_{1}}{\varphi_{0}}$ generates the function field of $\overline{\mathbb{H}^{2} / \Gamma(k)}$ over $\overline{\mathbb{H}^{2} / \Gamma}$. Furthermore, $w$ satisfies an equation of the form

$$
\prod_{j=1}^{k n(k)}\left(w-a_{j}\right)=0
$$

where $a_{j} \in \mathbb{C}(\jmath)$.

\section{Equations that are satisfied by the embedding.}

Let $k$ be an odd integer. Having mapped $\mathbb{H}^{2} / \Gamma(k)$ into $\mathbf{P} \mathbb{C}^{\frac{k-3}{2}}$, we would like to find the equations which define the image. In this section we will obtain a method for deriving such equations and then use the method to obtain some quartic relations between the functions defining the mapping. 


\subsection{The residue theorem.}

The next theorem is proved just as in [3].

Theorem 15.1. Let $\tau \in \mathbb{H}^{2}$. Let $f$ be a holomorphic function on the plane $\mathbb{C}$ which satisfies the two functional equations:

$$
f(z+1)=-f(z), z \in \mathbb{C},
$$

and

$$
f(z+\tau)=-\exp 2 \pi \imath\left\{-k z-k \frac{\tau}{2}\right\} f(z), z \in \mathbb{C}
$$

Then

$$
\sum_{l=0}^{k-1}(-1)^{l} \exp \pi \imath\left\{\frac{l^{2}}{k} \tau\right\} f\left(\frac{l}{k} \tau\right)=0
$$

Proof. The main observation is the following lemma.

Lemma 15.2. For each $\tau \in \mathbb{H}^{2}$, the function on $\mathbb{C}$

$$
z \mapsto \theta\left[\begin{array}{l}
1 \\
1
\end{array}\right]\left(z, \frac{\tau}{k}\right)
$$

satisfies the above two functional equations and vanishes to order one at all the lattice points $\left\{n+m \frac{\tau}{k} ; n, m \in \mathbb{Z}\right\}$ (and only at these points); hence in particular at the $k$ points $\left\{\frac{l}{k} \tau, l=0, \ldots, k-1\right\}$.

Proof. The functional equations follow at once from [7, (1)] (equation (3.5) above). The vanishing claim follows from the fact that the theta function with odd integral characteristic vanishes only at the lattice points (we are using the lattice generated by 1 and $\frac{\tau}{k}$ ). Note that for the lattice generated by 1 and $\tau$, this function has precisely $k$ inequivalent zeros located at the points listed above.

Returning to the proof of the theorem,

$$
z \mapsto g(z)=\frac{f(z)}{\theta\left[\begin{array}{l}
1 \\
1
\end{array}\right]\left(z, \frac{\tau}{k}\right)}, z \in \mathbb{C},
$$


is an elliptic function with periods $1, \tau$ with (at most) simple poles at the points in our list. The sum of the residues of an elliptic function in a period parallelogram must vanish; so we have

$$
\sum_{l=0}^{k-1} \operatorname{Res}_{\frac{l}{k} \tau} g=0 .
$$

Proceeding as in [3], we see that for each $l$,

$$
\operatorname{Res}_{\frac{l}{k} \tau} g=\frac{f\left(l \frac{\tau}{k}\right)}{\theta^{\prime}\left[\begin{array}{l}
1 \\
1
\end{array}\right]\left(l \frac{\tau}{k}, \frac{\tau}{k}\right)},
$$

where as usual ' $=\frac{\partial}{\partial z}$ (provided the denominator in the above expression does not vanish). Using once again (3.5) or [7, (1)], we see that

$$
\theta^{\prime}\left[\begin{array}{l}
1 \\
1
\end{array}\right]\left(l \frac{\tau}{k}, \frac{\tau}{k}\right)=\exp \pi \imath\left\{-l-l^{2} \frac{\tau}{k}\right\} \theta^{\prime}\left[\begin{array}{l}
1 \\
1
\end{array}\right]\left(0, \frac{\tau}{k}\right) .
$$

The observation that

$$
\theta^{\prime}\left[\begin{array}{l}
1 \\
1
\end{array}\right]\left(0, \frac{\tau}{k}\right) \neq 0
$$

(see, for example, [2]) completes the proof of the theorem. (A similar argument was used in [3].)

\subsection{The algorithm.}

We describe a general method (consisting of three steps) for obtaining theta identities among sums of products of theta constants of the form $\theta\left[\begin{array}{c}i \\ k \\ 1\end{array}\right]$ (with variable $i \in \mathbb{Z}$ and $k$ fixed and odd).

Step 0. Start with the following set of characteristics $\left[\begin{array}{c}\frac{i}{k} \\ 1\end{array}\right]$, where $i \in \mathbb{Z}$, $-k+2 \leq i \leq k$, and $i$ is odd. Denote this set by $\mathbb{X}$. Note that $|\mathbb{X}|=k$. To simplify notation, we shall abuse language and identify the characteristic $\left[\begin{array}{c}\frac{i}{k} \\ 1\end{array}\right]$ with the integer $i$.

Step 1. Choose $m \in \mathbb{Z}, 1<m<k$. Pick $l_{r} \in \mathbb{Z}$ such that $1 \leq r \leq m$ and $\sum_{r=1}^{m} l_{r} \equiv 0 \bmod k$. Without loss of generality (see next displayed equation), we may and hence do assume that $0 \leq l_{r}<k$ for each $r$. 
Step 2. For each $r, 1 \leq r \leq m$, delete from $\mathbb{X}$ the unique characteristic $\left[\begin{array}{c}\frac{k_{r}}{k} \\ 1\end{array}\right]$ for which

$$
\theta\left[\begin{array}{c}
\frac{k_{r}}{k} \\
1
\end{array}\right]\left(\frac{l_{r}}{k} \tau, \tau\right)=0
$$

This means that

$$
k_{r}=k-2 l_{r} .
$$

Denote the deleted set by $\mathbb{A}$. From the characteristics in $\mathbb{X}-\mathbb{A}$, we choose ${ }^{20}$ $m$ characteristics $\alpha_{r}$ with the property that $\sum_{r=1}^{m} \alpha_{r} \equiv 0 \bmod k$. The $\alpha_{r}$ 's are not necessarily distinct. We define two functions on $\mathbb{C}$ :

$$
g(z)=\prod_{r=1}^{m} \theta\left[\begin{array}{c}
\frac{\alpha_{r}}{k} \\
1
\end{array}\right](z, \tau),
$$

and

$$
f(z)=\prod_{j \in \mathbb{X}-\mathbb{A}} \theta\left[\begin{array}{c}
\frac{j}{k} \\
1
\end{array}\right](z, \tau) g(z) .
$$

A straightforward calculation (using (3.5)) tells us that the function $f$ satisifies the conditions of the last theorem (because $\sum_{r=1}^{m}\left(k_{r}-\alpha_{r}\right) \equiv 0 \bmod 2 k$ ), and vanishes at $k-m$ points $\left\{\frac{j}{k} \tau ; j \in \mathbb{Z}, 0 \leq j<k, j \neq l_{r}\right\}$. Hence the theorem yields an identity involving a sum of $m$ products of $k$ theta functions evaluated at proper points. (There may, of course, be the possibility of lots of cancellation, and we can by changing the variable and the characteristic replace theta functions by theta constants, as we do in the next subsection.)

\subsection{Three term identities.}

We use the $(m=) 3$ step algorithm outlined above to produce 3 term identities. Choose $l_{1}=0, l_{2}=j, l_{3}=k-j$, with $j \in \mathbb{Z}, 0<j \leq \frac{k-1}{2}$. The characteristics to be deleted by step 2 are: $\left[\begin{array}{l}1 \\ 1\end{array}\right],\left[\begin{array}{c}\frac{-k+2 j}{k} \\ 1\end{array}\right],\left[\begin{array}{c}\frac{k-2 j}{k} \\ 1\end{array}\right]$. From the remaining set of $k-3$ characteristics, we choose 3 characteristics

\footnotetext{
${ }^{20}$ We do not know if such a choice is always possible. However, if $l_{r} \neq \frac{k-1}{2}$, $l_{r} \neq \frac{m-1}{2}$ for odd $m$, and $l_{r} \neq \frac{k+m-1}{2}$ for even $m$ (this means that $k_{r} \neq 1$, $k_{r} \neq k-(m-1)$ for odd $m$, and $k_{r} \neq 1-m$ for even $m$ ), then we can choose $\alpha_{i}=1$ for $i=1, \ldots, m-1, \alpha_{m}=k-(m-1)$ for odd $m$, and $\alpha_{m}=-(m-1)$ for even $m$. This prescription could lead to trivial identities, for example if $m=2$.
} 
$\alpha_{r}, r=1,2,3$, such that $\sum_{i=1}^{3} \alpha_{r} \equiv 0 \bmod k$. The function $f$ is defined as

$$
f(z)=\prod_{i \in \mathbb{X}} \theta^{n_{i}}\left[\begin{array}{l}
\frac{i}{k} \\
1
\end{array}\right](z, \tau),
$$

where $n_{i} \in \mathbb{Z}$ for $i \in \mathbb{X}$ is computed as $n_{i}=1+$ the number of $r$ for which $\alpha_{r}=i$ if $i \neq-k+2 j, k-2 j, k$ and $n_{i}=0$ if $i=-k+2 j, k-$ $2 j$, or $k$. We need to calculate the values of the function $f$ at the 3 points $0, \frac{j}{k} \tau$, and $\frac{k-j}{k} \tau$. The last theorem therefore yields

$$
\begin{gathered}
\prod_{i \in \mathbb{X}, i \neq k,-k+2 j, k-2 j} \theta^{n_{i}}\left[\begin{array}{c}
\frac{i}{k} \\
1
\end{array}\right](0, \tau) \\
+(-1)^{j} \exp \pi \imath\left\{\frac{j^{2}}{k} \tau\right\} \prod_{i \in \mathbb{X}, i \neq k,-k+2 j, k-2 j} \theta^{n_{i}}\left[\begin{array}{c}
\frac{i}{k} \\
1
\end{array}\right]\left(\frac{j}{k} \tau, \tau\right) \\
+(-1)^{k-j} \exp \pi \imath\left\{\frac{(k-j)^{2}}{k} \tau\right\} \prod_{i \in \mathbb{X}, i \neq k,-k+2 j, k-2 j} \theta^{n_{i}}\left[\begin{array}{c}
\frac{i}{k} \\
1
\end{array}\right]\left(\frac{k-j}{k} \tau, \tau\right)=0 .
\end{gathered}
$$

Using (3.6), we change the above formula to a relation among only theta constants:

$$
\begin{gathered}
\prod_{i \in \mathbb{X}, i \neq k, k-2 j, 2 j-k} \theta^{n_{i}}\left[\begin{array}{c}
\frac{i}{k} \\
1
\end{array}\right](0, \tau)+\prod_{i \in \mathbb{X}, i \neq k, k-2 j, 2 j-k} \theta^{n_{i}}\left[\begin{array}{c}
\frac{i+2 j}{k} \\
1
\end{array}\right](0, \tau) \\
+\prod_{i \in \mathbb{X}, i \neq k, k-2 j, 2 j-k} \theta^{n_{i}}\left[\begin{array}{c}
\frac{i-2 j}{k} \\
1
\end{array}\right](0, \tau)=0 .
\end{gathered}
$$

The terms involving the exponential function miraculously canceled out because

$$
\sum_{i \in \mathbb{X}} n_{i}=k
$$

The last formula for the identity can be reduced by use of (3.1) and by (3.2) to one involving only the characteriscs

$$
\{1,3, \ldots, k-2\} \text {. }
$$

This involves introducing some $2 k$-th roots of unity and leads to cancellation of most terms. We start with the observation that for any integer $l$ translation of the characteristics in $\mathbb{X}$ by the characteristic $\left[\begin{array}{c}\frac{2 l}{k} \\ 1\end{array}\right]$ produces 
an automorphism of $\mathbb{X}$, provided addition is interpreted modulo $2 k$, that as a consequence of (3.2) does not otherwise alter any of the above formula. This reduces the set of characteristics in the formula for the identity to

$$
\{-k+2, \ldots,-1,1,3, \ldots, k\} \text {. }
$$

Next, using (3.1) and (3.2), we replace the term $\theta\left[\begin{array}{c}\frac{l}{k} \\ 1\end{array}\right]$ (where $l$ is an odd integer with $-k+2 \leq l \leq-1)$ by the term $\exp \left(\pi \imath \frac{l}{k}\right) \theta\left[\begin{array}{c}\frac{-l}{k} \\ 1\end{array}\right]$. We have reduced the set of characteristics appearing in our formulae to

$$
\{1,3, \ldots, k\} \text {. }
$$

Our final observation is that the characteristic $k$ never appears. We then proceed to cancel common terms. We have obtained

Theorem 15.3. Let $j \in \mathbb{Z}, 0<j \leq \frac{k-1}{2}$. For $i=1,2,3$, choose odd integers $\alpha_{i}$ with $\left|\alpha_{i}\right| \leq k-2,\left|\alpha_{i}\right| \neq k-2 j$, and $\sum_{i=1}^{3} \alpha_{i} \equiv 0 \bmod k$. The following quartic relation holds among theta constants:

$$
\begin{gathered}
\theta\left[\begin{array}{c}
\frac{k-4 j}{k} \\
1
\end{array}\right] \prod_{i=1,2,3} \theta\left[\begin{array}{c}
\frac{\alpha_{i}}{k} \\
1
\end{array}\right]+\exp \left(\pi \imath \frac{k-4 j}{k}\right) \theta\left[\begin{array}{c}
\frac{k-2 j}{k} \\
1
\end{array}\right] \prod_{i=1,2,3} \theta\left[\begin{array}{c}
\frac{\alpha_{i}+2 j}{k} \\
1
\end{array}\right] \\
+\exp \left(\pi \imath \frac{-k+2 j}{k}\right) \theta\left[\begin{array}{c}
\frac{k-2 j}{k} \\
1
\end{array}\right] \prod_{i=1,2,3} \theta\left[\begin{array}{c}
\frac{\alpha_{i}-2 j}{k} \\
1
\end{array}\right]=0 .
\end{gathered}
$$

Problem 3. For fixed $k$, the above identity is determined by the quadruple $\left(j ; \alpha_{1}, \alpha_{2}, \alpha_{3}\right)$. It is obvious that both $\left(j ; \alpha_{1}, \alpha_{2}, \alpha_{3}\right)$ and $\left(j ;-\alpha_{1},-\alpha_{2},-\alpha_{3}\right)$ always determine the same identity. There are other, less clear, relations among the identities. For $k=9$, for example, the quadruples $(1 ; 1,3,5)$ and $(2 ;-7,-3,1)$ lead to the same identity. It is of interest to determine minimal generators for the ideal of identities and whether the identities determine the curve $\Phi\left(\overline{\mathbb{H}^{2} / \Gamma(k)}\right) \subset \mathbf{P} \mathbb{C}^{\frac{k-3}{2}}$.

\subsection{Examples of equations.}

We conclude this section by constructing explicit examples for the cases $k=7,9$. We define ${ }^{21} \theta_{l}=\theta\left[\begin{array}{c}\frac{2 l+1}{k} \\ 1\end{array}\right]$ for $l=0,1, \ldots, \frac{k-3}{2}$. For $k=7$, we

\footnotetext{
${ }^{21}$ Obviously the functions $\theta_{l}$ and $\varphi_{l}$ are closely related. We add this definition to emphasize that an identity among theta constants in the variable $k \tau$ (or $\frac{\tau}{k}$ ) is
} 
choose $j=1, \alpha_{1}=\alpha_{2}=3$, and $\alpha_{3}=1$. From the last theorem we obtain

Theorem 15.4. We have for $k=7$,

$$
\theta_{0}^{3} \theta_{2}+\epsilon^{9} \theta_{2}^{3} \theta_{1}+\epsilon^{6} \theta_{1}^{3} \theta_{0}=0, \epsilon=\exp \left(\frac{\pi \imath}{7}\right) .
$$

Remark 14. We have reproven (10.1).

We consider next the case $k=9$. We have seen that $\mathbb{H}^{2} / \Gamma(9)$ is a closed surface of genus 10 punctured at 36 points. The compact surface $\overline{\mathbb{H}^{2} / \Gamma(9)}$ is mapped into $\mathbf{P} \mathbb{C}^{3}$ by $\Phi=\left(\varphi_{0}, \varphi_{1}, \varphi_{2}, \dot{\varphi}_{3}\right)$. Omitting the function $\varphi_{1}$ we obtain a mapping of the surface $\mathbb{H}^{2} /<\Gamma(9), B^{3}>$ into $\mathbf{P} \mathbb{C}^{2}$. We seek the equation of the image; that is, equations among the $\varphi_{l}$ (equivalently among $\left.\theta_{l}\right), l \neq 1$. Choosing $j=3, \alpha_{1}=\alpha_{2}=1, \alpha_{3}=7$, we get the equation

$$
\theta_{0}^{2} \theta_{3}+\epsilon^{13} \theta_{3}^{2} \theta_{2}+\epsilon^{8} \theta_{2}^{2} \theta_{0}=0, \epsilon=\exp \left(\frac{\pi \imath}{9}\right) .
$$

This is (with each $\theta$ replaced by a $\varphi$ ) a nonsingular equation of a torus; the torus $\overline{\mathbb{H}^{2} /<\Gamma(9), B^{3}>}$ sitting in $\mathbf{P} \mathbb{C}^{2}$. The zero set of this equation is irreducible (hence connected); see for example, [14, p. 27].

We obtain equations involving the rest of the characterestics by choosing $j=1,2,4$ respectively and $\alpha_{1}=\alpha_{2}=\alpha_{3}=3$ :

$\theta_{2} \theta_{1}^{3}+\epsilon^{5} \theta_{3}\left(\theta_{2}^{3}+\epsilon^{6} \theta_{0}^{3}\right)=0, \theta_{0} \theta_{1}^{3}+\epsilon \theta_{2}\left(\theta_{3}^{3}-\theta_{0}^{3}\right)=0, \theta_{3} \theta_{1}^{3}-\theta_{0}\left(\epsilon^{6} \theta_{3}^{3}+\theta_{2}^{3}\right)=0$.

We can however use $\psi_{l}$ instead of $\varphi_{l}$ to map our surface into projective space. As a consequence of (4.7) we are led to the same identities in terms of $\varphi_{l}$ and in terms of $\psi_{l}$. The introduction of the change of variable

$$
x_{l}=\exp \left\{-\frac{\pi \imath l}{9}\right\} \psi_{l}, l=0, \ldots, 3,
$$

leads to more elegant formulae (with \pm 1 as coefficients) and the

Theorem 15.5. The intersection of the following 5 hypersurfaces in $\mathbf{P} \mathbb{C}^{3}$ define the surface $\overline{\mathbb{H}^{2} / \Gamma(9)}$ :

$$
\begin{aligned}
& x_{0}^{2} x_{3}+x_{3}^{2} x_{2}-x_{2}^{2} x_{0}=0,-x_{0} x_{1}^{3}+x_{2}\left(x_{3}^{3}+x_{0}^{3}\right)=0 \\
& -x_{2} x_{1}^{3}+x_{3}\left(x_{2}^{3}+x_{0}^{3}\right)=0,-x_{3} x_{1}^{3}+x_{0}\left(x_{2}^{3}-x_{3}^{3}\right)=0 \\
& x_{1}^{9}-x_{2}^{3} x_{0}^{6}+x_{3}^{3} x_{0}^{6}-x_{3}^{3} x_{2}^{6}+x_{2}^{3} x_{3}^{6}+x_{0}^{3} x_{3}^{6}-x_{0}^{3} x_{2}^{6}=0 .
\end{aligned}
$$

equivalent to an identity for the variable $\tau$. 
Proof. The first 4 relations are the translates to the $x_{l}$ variables of our last 4 three term identities (given in the $\theta_{l}$ variables). The last relation is, of course, not a three term identity. It is obtained through the use of the residue theorem. More precisely, we use the fact that the sum of the residues of the elliptic function (for fixed $\tau \in \mathbb{H}^{2}$ )

$$
z \mapsto \frac{\theta^{3}\left[\begin{array}{c}
1 \\
\frac{3}{9}
\end{array}\right](z, \tau) \theta^{6}\left[\begin{array}{c}
1 \\
\frac{15}{9}
\end{array}\right](z, \tau)}{\theta\left[\begin{array}{l}
1 \\
1
\end{array}\right](9 z, 9 \tau)}, z \in \mathbb{C},
$$

is zero. The point $(0,1,0,0) \in \mathbf{P} \mathbb{C}^{3}$ which does not belong to $\Phi\left(\overline{\mathbb{H}^{2} / \Gamma(9)}\right)$ will satisfy every three term identity derived from Theorem 15.3. Hence another type of identity is needed to describe the curve $\Phi\left(\overline{\mathbb{H}^{2} / \Gamma(9)}\right)$ in $\mathbf{P} \mathbb{C}^{3}$. The fifth equation serves this purpose.

Denote by $H^{0}$ the set defined by the first equation in $\mathbf{P} \mathbb{C}^{2}$, and by $H^{1}$ the set in $\mathbf{P} \mathbb{C}^{3}$ defined by the entire set of 5 equations. We have already observed

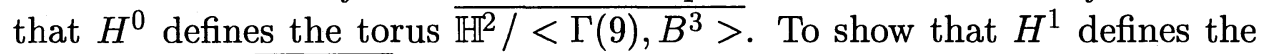
closed surface $\overline{\mathbb{H}^{2} / \Gamma(9)}$, we study the commutative diagram where $\pi$ is the canonical projection of $\overline{\mathbb{H}^{2} / \Gamma(9)}$ onto $\overline{\mathbb{H}^{2} / \Gamma(9)} /\left\langle\tilde{B}^{3}\right\rangle$,

$$
\begin{gathered}
X(\tau)=\left(x_{0}(\tau), x_{1}(\tau), x_{2}(\tau), x_{3}(\tau)\right), \tau \in \mathbb{H}^{2}, \\
X_{1}(\tau)=\left(x_{0}(\tau), x_{2}(\tau), x_{3}(\tau)\right), \tau \in \mathbb{H}^{2},
\end{gathered}
$$

and

$$
\omega\left(x_{0}, x_{1}, x_{2}, x_{3}\right)=\left(x_{0}, x_{2}, x_{3}\right),\left(x_{0}, x_{1}, x_{2}, x_{3}\right) \in \mathbf{P} \mathbb{C}^{3}-\{(0,1,0,0)\} .
$$

The map $\omega$ is well defined since its domain excludes the point $(0,1,0,0)$. We already pointed out that the image of the map $X$ avoids this point. We know that $\operatorname{deg} \pi=3, X$ is injective, and that

$$
X\left(\overline{\mathbb{H}^{2} / \Gamma(9)}\right) \subset H^{1} .
$$

The proof of the theorem can now be completed by showing that every point $x \in H^{0}$ has (at most) 3 preimages (counting multiplicities) in $H^{1}$. If the $i$-th component of $x$ is nonzero, then equation $i+1$ shows that $\omega^{-1}(x)$ has 3 preimages in $H^{1}$ (counting multiplicity). It is easier to deal with set theoretic preimages. The primage in $\mathbf{P} \mathbb{C}^{3}-\{(0,1,0,0)\}$ under $\pi$ of a point $x=\left(x_{0}, x_{2}, x_{3}\right) \in \mathbf{P} \mathbb{C}^{2}$ consists of either 3 distinct points or a single point. If at least one of the 3 pairs

$$
\left(x_{0}, x_{2}\left(x_{3}^{3}+x_{0}^{3}\right)\right),\left(x_{2}, x_{3}\left(x_{2}^{3}+x_{0}^{3}\right)\right),\left(x_{3}, x_{0}\left(x_{2}^{3}-x_{3}^{3}\right)\right)
$$


has 2 nonzero entries, then $\pi^{-1}\left(x_{0}, x_{2}, x_{3}\right)$ contains 3 distinct points. The points

$$
(1,0,0),(0,1,0) \text { and }(0,0,1)
$$

belong to $H^{0}$; their (unique) preimages in $H^{1}$ are the points

$$
(1,0,0,0),(0,0,1,0) \text { and }(0,0,0,1),
$$

respectively. The remaining points $x=\left(x_{0}, x_{2}, x_{3}\right)$ in $H^{0}$ have 3 nonzero components. Thus it involves no loss of generality to assume that $x_{0}=-1$. For the preimage of such an $x$ to consist of a single point, we must have that both $x_{2}$ and $x_{3}$ are cube roots of unity. We conclude there are at most 9 points (in addition to the coordinate vectors) in $H^{1}$ which are preimages of branch values of the map $\pi$; that is, points of the form

$$
\left(-1, \eta^{j}, \eta^{l}\right), \eta=\exp \frac{2 \pi \imath}{3}, j, l=0,1,2 .
$$

However, not all 9 points belong to $H^{0}$. Only the 6 pairs $(j, l)=(0,1),(0,2)$, $(1,0),(1,1),(2,0)$, and $(2,2)$ correspond to points in $H^{0}$. We have shown that with 9 exceptions (corresponding to the punctures on $\left(\mathbb{H}^{2} / \Gamma(9)\right) /<$ $\tilde{B}^{3}>$ ) the preimage of a point in $H^{0}$ contains precisely 3 points of $H^{1}$. The only remaining possibility is for $H^{1}$ to contain the point $(0,1,0,0)$. This possibility is eliminated by the fifth equation.

Corollary 15.6. The map $X$ is injective and of maximal rank. Hence its image is a nonsingular irreducible subvariety of $\mathbf{P} \mathbb{C}^{3}$.

Proof. In the proof of the Theorem we already used that $X$ is injective; it is of maximal rank because $X_{1}$ is. The image of $X$ is nonsingular by Chow's theorem (see, for example, [9, pg. 167]); it is irreducible by the results of the theorem or as a consequence of the fact that it is the image under $X$ of a compact Riemann surface.

Remark 15. If we are willing to use algebraic sets AND their complements, then we can get much simpler description of the surface $\overline{\mathbb{H}^{2} / \Gamma(9)} \subset \mathbf{P} \mathbb{C}^{3}$ as the set of those $X=\left(x_{0}, x_{1}, x_{2}, x_{3}\right) \in \mathbf{P} \mathbb{C}^{3}$ that satisfy the first 4 equations of the theorem and $X \neq(0,1,0,0)$.

We have already observed that $\overline{\mathbb{H}^{2} / \Gamma(13)}$ is a closed surface of genus 50 . It is convenient to work with the map

$$
X=\left(x_{0}, \ldots, x_{5}\right): \overline{\mathbb{H}^{2} / \Gamma(13)} \rightarrow \mathbf{P} \mathbb{C}^{5},
$$


where

$$
x_{l}=\exp \left\{-\frac{\pi \imath l}{13}\right\} \psi_{l}, l=0, \ldots, 5 .
$$

We define a map from

$$
\mathbb{Y}=\left\{\left(x_{0}, \ldots, x_{5}\right) \in \mathbf{P} \mathbb{C}^{5} ;\left(x_{0} x_{2}, x_{1} x_{5}, x_{3} x_{4}\right) \neq 0\right\}
$$

to $\mathbf{P} \mathbb{C}^{2}$ by $\Omega\left(x_{0}, x_{1}, x_{2}, x_{3}, x_{4}, x_{5}\right)=\left(x_{0} x_{2}, x_{1} x_{5}, x_{3} x_{4}\right)=X_{1}$. It is easy to see that $\left(x_{0} x_{2}, x_{1} x_{5}, x_{3} x_{4}\right)$ is invariant under $B$ and $\gamma=\left[\begin{array}{cc}5 & -2 \\ 13 & -5\end{array}\right]$; moreover from Theorem 15.3, we obtain (by setting $j=1, \alpha_{1}=1, \alpha_{2}=$ $\left.5, \alpha_{3}=7\right)$ the identity $\left(x_{0} x_{2}\right)\left(x_{1} x_{5}\right)+\left(x_{1} x_{5}\right)\left(x_{3} x_{4}\right)-\left(x_{3} x_{4}\right)\left(x_{0} x_{2}\right)=0$. The equation gives a quadratic curve in $\mathbf{P} \mathbb{C}^{2}$. It is well known that a nonsingular quadratic curve is a Riemann surface of genus 0 (the result also follows from $\S 12)$. Even though $X\left(\overline{\mathbb{H}^{2} / \Gamma(13)}\right) \not \subset \mathbb{Y}$, we have obtained a projective embedding $X_{1}: \overline{\mathbb{H}^{2} /(<G(13), \gamma>)} \rightarrow \mathbf{P} \mathbb{C}^{2}$. We have seen in $\S 12$, that $\overline{\left.\mathbb{H}^{2} /<G(13), \gamma\right\rangle}$ is conformally equivalent to $\mathbf{P} \mathbb{C}$.

\section{References.}

[1] J.H. Conway, The Atlas of Finite Simple Groups, Oxford Clarendon Press, 1985.

[2] H.M. Farkas, Elliptic functions and modular forms, Contributions to Analysis, Academic Press, 1974, pp. 133-145.

[3] H.M. Farkas and Y. Kopeliovich, New theta constant identities, Israel J. Math. 82 (1993), 133-140.

[4] (1995), 1009-1020.

[5] H.M. Farkas and I. Kra, Automorphic forms for subgroups of the modular group. II: Composite level, submitted.

[6] , Riemann Surfaces (second edition), Graduate Texts in Mathematics, vol. 71, Springer-Verlag, 1992.

[7] Automorphic forms for subgroups of the modular group, Israel J. Math. 82 (1993), 87-131.

[8] R. Fricke and F. Klein, Vorlesungen über die Theorie der elliptischen Modulfunktionen, B.G. Teubner, 1890-2. 
[9] P. Griffiths and J. Harris, Principles of Algebraic Geometry, John Wiley \& Sons, 1978.

[10] G.H. Hardy and E.M. Wright, An Introduction to the Theory of Numbers, Oxford University Press, 1960.

[11] J.E. Humphreys, Representations of $S L(2, p)$, Amer. Math. Monthly 82 (1975), 21-39.

[12] F. Klein, Uber die elliptischen Normalcurven der $n$-ten ordenung, Felix Klein Gesammelte Mathematishe Abhandulgen (volume 3), Verlag von Julius Springer, 1923, pp. 199-254.

[13] Uber gewisse teilwerte der theta funktionen, Felix Klein Gesammelte Mathematishe Abhandulgen (volume 3), Verlag von Julius Springer, 1923, pp. 186-197.

[14] A.W. Knapp, Elliptic Curves, Mathematical Notes, vol. 40, Princeton University Press, 1992.

[15] S. Lang, Algebra, Addison-Wesley, 1971.

[16] T. Miyake, Modular Forms, Springer-Verlag, 1989.

[17] H.E. Rauch and J. Lewittes, The Riemann surface of Klein with 168 automorphisms, Problems in Analysis, Princeton University Press, 1970, pp. 297-308.

[18] C.-H. Sah, Groups related to compact Riemann aurfaces, Acta Math. 123 (1969), 13-42.

[19] J.-P. Serre, A Course in Arithmetic, Graduate Texts in Mathematics, vol. 7, Springer-Verlag, 1973.

[20] G. Shimura, Introduction to the Arithmetic Theory of Automorphic Functions, Publications of the Mathematical Society of Japan, Kanô memorial lectures 1, vol. 11, Iwannami Shoten and Princeton University Press, 1971.

The Hebrew University OF Jerusalem

JERUSALEM, ISRAEL

University of CALIFoRNia AT IRVINE

IRVINE, CA 92717

AND

State University of NeW York at Stony Brook

STONY BROOK, NY 11794 
ReCeived MaRCH 29Th, 1995. 